\title{
Magnetoelastic stresses in rare-earth thin films and superlattices
}

\author{
J. I. Arnaudas, M. Ciria, C. de la Fuente, L. Benito, and A. del Moral \\ Departamento de Magnetismo de Sólidos, Departamento de Fisica de la Materia Condensada and ICMA, \\ Universidad de Zaragoza and CSIC, 50071 Zaragoza, Spain \\ E-mail: arnaudas@posta.unizar.es \\ R. C. C. Ward and M. R. Wells \\ Dept. of Physics, Clarendon Laboratory, Oxford OX1 3PU, U.K.
}

\section{Dufour, K. Dumesnil, and A. Mougin}

Laboratoire de Métallurgie Physique et de Science des Matériaux, Université Henri Poincaré, Nancy, France

Received October 26, 2000

\begin{abstract}
We report on the study of the magnetoelastic behavior of some rare-earth based thin films and superlattices (SL's). Magnetoelastic stress (MS) measurements (by using a cantilever capacitive technique) within a wide range of temperatures $(10-300 \mathrm{~K}$ ) and magnetic fields (up to $12 \mathrm{~T}$ ) have been performed. We derive expressions relating the cantilever curvatures and the magnetoelastic stresses in anisotropic thin films and SL's (for cubic symmetry) deposited onto crystalline substrates. The magnetoelastic energy associated to the interfaces and the epitaxial stress dependence of the volume MS has been investigated by studying the basal plane $\mathrm{MS}$ in $\mathrm{Ho}_{n} / \mathrm{Lu}_{15}$ and in $\mathrm{Ho}_{10} \mathrm{Y}_{m}$ SL's: we obtain interface MS even higher than the volume ones and the effect in bulk's MS of the epitaxial strain is large. In $\mathrm{Dy} / \mathrm{Y}$ and $\mathrm{Er} / \mathrm{Lu}$ SL's we also deduce the MS contributions but, for $\mathrm{Er} / \mathrm{Lu}$, incomplete saturation leads to inconclusive results. Although the latter case also happens in Ho/Tm SL's, MS clearly shows anisotropy competition. In $\mathrm{TbFe}_{2}(t) / \mathrm{YFe}_{2}(1000 \AA)(300 \AA<t<1300 \AA$ ) epitaxial bilayers, we determine all the MS allowed by the symmetry and show that epitaxial stress strongly modifies the tetragonal MS. The thermal dependence of MS parameters is also analysed.
\end{abstract}

PACS: 75.70.-i, 75.80.+q

\section{Introduction}

The study of the magnetoelastic (ME) properties of magnetic thin films and layered nanostructures, where magnetic layers are interleaved by non-magnetic ones or by other having different magnetic properties, presents considerable interest, not only because of fundamental reasons, but also due to the technological applications of these artificial systems. For example, the success of molecular beam epitaxy (MBE) of rare-earth (RE) superlattices (SL's) has allowed the investigation of the novel magnetic properties exhibited by the well known $\mathrm{RE}$ metals when thin layers of a rare earth are interleaved with non-magnetic layers (e.g., Lu or Y) or with layers made from a different rare earth.
Although much research has been performed to date in relation with different magnetic properties [1-3] of these nanostructures, it was only recently that direct magnetoelastic stress measurements were performed in RE-based superlattices [4-6]. The knowledge of the magnetoelastic behavior of RE SL's is important because of the influence of the ME energy in the spin configuration and magnetic properties of such systems. Several RE/Y and RE/Lu SL's have been studied and some of the differences in the magnetic behavior observed on having $\mathrm{Y}$ or $\mathrm{Lu}$ as interleaving layers are currently attributed to the different epitaxial strain, tensile or compressive, respectively, occuring in both cases. For instance, in bulk Ho the basal plane ME energy is one of the 
agents driving the magnetic structure from helical to a ferro-cone phase [7]; interestingly, in $\mathrm{Ho} / \mathrm{Y}$ SL's this ferro-cone phase is suppressed, unlike in $\mathrm{Ho} / \mathrm{Lu}$ systems, where the effect of the compressive stress stabilizes the ferromagnetic phase $[3,8]$, the FM transition temperature increasing for decreasing Ho fraction. Regarding the RE SL's, in this paper we will review the magnetoelastic stress studies we carried out in two series of $\mathrm{Ho} / \mathrm{Lu}$ and $\mathrm{Ho} / \mathrm{Y}$ superlattices, as well as in $\mathrm{Dy} / \mathrm{Y}$ and $\mathrm{Er} / \mathrm{Lu} \mathrm{SL}$ 's and in the more complex SL system, $\mathrm{Ho} / \mathrm{Tm}$.

On the other hand, and concerning nanostructured systems suitable for applications, some RE alloys are, in principle, the logic choice. So, new devices, such as microsystems actuators working at room temperature, should be based on alloys with very large magnetostriction at moderately low fields. The well-known $\mathrm{REFe}_{2}$ (RE: rare earth or rare-earth alloy) Laves phases appear as good candidates to fulfil the necessary requirements for applications. The magnetostriction of bulk single-crystal and polycrystalline $\mathrm{REFe}_{2}$ was thoroughly studied in the 1970's and a noticeable effort has been made in the last years to produce amorphous and polycrystalline RE-TM (TM: transition metal) films and spring-magnet type multilayers of with improved magnetoelastic properties $[9,15]$. However, the intrinsic magnetoelastic behavior of thin-film samples had not been compared with that of crystalline bulk materials due to the lack of $\mathrm{REFe}_{2}$ single-crystal films. The recent success in growing by MBE high quality epitaxial films of these Laves phase magnets [16] has opened the possibility of perform such kind of studies $[17,18]$. In this article we present magnetoelastic stress measurements performed in a series of $\mathrm{TbFe}_{2} / \mathrm{YFe}_{2}$ (110) single-crystal bilayers.

First of all we will describe in detail the foundations of the experimental technique employed, a cantilever method. This is important because the analysis of the cantilever deflections, in the case of crystalline films and substrates, differs markedly from that performed for isotropic plates, from which it is obtained the following expression relating the cantilever deflection and the magnetostriction $[19,20]$ :

$$
\Delta=3 \lambda_{s} h_{m s}\left(\frac{L}{h_{\text {subs }}}\right)^{2} \frac{E_{m s}}{E_{\text {subs }}} \frac{\left(1+v_{\text {subs }}\right)}{\left(1+v_{m s}\right)},
$$

where $\lambda_{\text {subs }}$ is the magnetostriction; $L$, the length of the plate; $h_{\text {subs }}$ and $h_{m s}$, the substrate and film thicknesses, respectively; $E$ and $v$ are the Young moduli and Poisson ratios; the subscripts $m s$ and subs refer to film and substrate, respectively. For films or SL's having hexagonal symmetry an adequate formula, expressed in terms of the magnetoelastic stresses $B_{p}^{\mu l}$, instead of in terms of the magnetostriction coefficients $\lambda_{j k}^{\mu}$, has been derived [4,21]. The case of anisotropic substrates and the boundary conditions imposed by the experimental situation, have also been considered [4] and will be outlined below for films having cubic symmetry. The analysis of the hexagonal symmetry case can be found elsewhere [6].

\section{Determination of magnetoelastic stresses in anisotropic thin films and superlattices: case of cubic symmetry}

In the following we deal with a situation slightly more complicated than the occurring in hexagonal systems with cylindrical symmetry. In the cubic case, to be able to determine all the relevant magnetoelastic stress parameters from the experiments, we should perform measurements with the sample cut along different crystalline directions, and consequently, we need to obtain the corresponding expressions relating the magnetoelastic stresses and the plate curvatures.

\subsection{Evaluation of the elastic energy of a thin plate}

We consider a thin plate where the length $l$ and width $w$ are much smaller than the thickness $h$ (in our samples $h$ is $10^{-2}$ times the other dimensions). Since, in our particular case, the samples were grown in the (110) plane, we have cut the samples using two types of configurations, which will be considered to get practical expressions. In the first configuration, our coordinate system axes $y^{\prime}$ and $z^{\prime}$ are parallel to the sample sides of dimensions $l_{1}$ and $w_{1}$, respectively, and $x^{\prime}$ axis is normal to the growing plane, being $y^{\prime}$ axis $\|$ [110], $z^{\prime}$ axis $\|[001]$ and $x^{\prime}$ axis $\|[110]$. In the second one, our coordinate system axes $y^{\prime \prime}$ and $z^{\prime \prime}$ are parallel to the new sides of dimensions $l_{2}$ and $w_{2}$, respectively, and $x^{\prime \prime}=x^{\prime}$, being $y^{\prime \prime}$ axis $\|[\overline{1} 11]$ and $z^{\prime \prime}$ axis $\|$ [11 2$]$. To save space, we will label the coordinate systems $O X^{\prime} Y^{\prime} Z^{\prime}$ as $S^{\prime}$ and $O X^{\prime \prime} Y^{\prime \prime} Z^{\prime \prime}$ as $S^{\prime \prime}$; also the axes and superscripts corresponding to both systems will be denoted with a single label, $s$, for a more compact notation, being $s={ }^{\prime}$ or ', respectively, unless otherwise specified. The boundary conditions on the plate's surfaces are $\sigma_{i k} n_{k}=P_{i}$, where $n_{k}$ are the components of the normal to the plate surface, $\sigma_{i k}$ are the stress tensor components and $P_{i}$ are the components of the external pressure applied to the plate. Because of the strength of the internal $\mathrm{co}^{-}$ 
hesive forces, much higher than the external forces, we can consider that $\sigma_{i^{s} x^{s}}=0\left(i^{s}=x^{S}, y^{s}\right.$ and $\left.z^{s}\right)$ on the plate surface. Within a thin plate, these components of the stress tensor are small compared with the other ones, and can also be neglected [22]. We will now assume a pure bending of the plate, i.e., bending without torsion; in this case, it is easy to show that the longitudinal strains are given by [23]

$$
\varepsilon_{y^{s} y^{s}}=\frac{x^{s}}{R_{y^{s}}}, \varepsilon_{z^{s} z^{s}}=\frac{x^{s}}{R_{z^{s}}}, \varepsilon_{y^{s} z^{s}}=0 .
$$

In Eq. (1), $x^{s}$ is the coordinate of the plane where the strains are evaluated $\left(x^{s}=0\right.$ is the neutral plane); $R_{i^{s}}\left(i^{s}=y^{s}, z^{s}\right)$ (with $s={ }^{\prime}$ and ") are the radii of the curvature of the neutral plane of the plate in the planes: $y^{\prime} x^{\prime}$ and $z^{\prime} x^{\prime}$ planes in the first case, and $y^{\prime \prime} x^{\prime \prime}$ and $z^{\prime \prime} x^{\prime \prime}$ planes for the second one. The elastic energy density for the plate in our both situations:

$$
\mathrm{e}_{\text {elas }}^{s}=\frac{1}{2} \sigma_{i j} \varepsilon_{i j} \varepsilon_{i s}
$$

is obtained by writing the stresses in terms of the

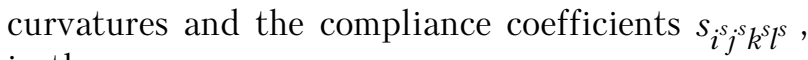
in the way

$$
\begin{aligned}
& \sigma_{y^{s} y^{s}}=\frac{x^{s}}{\left(\hat{s}_{1} 1_{1} \hat{s}_{2^{s} 2^{s}}-\hat{s}_{\left.1^{s} 2^{s}\right)}^{2}\right.}\left(\frac{\hat{s}_{2^{s} 2^{s}}}{R_{y^{s}}}-\frac{\hat{s}_{1^{s} 2^{s}}}{R_{z^{s}}}\right), \\
& \sigma_{z^{s} z^{s}}=\frac{x^{s}}{\left(\hat{s}_{1} 1_{1} s \hat{s}_{2^{s} 2^{s}}-\hat{s}_{\left.1^{s} 2^{s}\right)}^{2}\right.}\left(\frac{\hat{s}_{1^{s} 1^{s}}}{R_{z^{s}}}-\frac{\hat{s}_{1^{s} 2^{s}}}{R_{y^{s}}}\right),
\end{aligned}
$$

where

$$
\hat{s}_{1^{s} 1^{s}}=s_{1^{s} 1^{s}}-\frac{s_{1^{s} 6^{s}}^{2}}{s_{6^{s} 6^{s}}}, \hat{s}_{2^{s} 2^{s}}=s_{2^{s} 2^{s}}-\frac{s_{2^{s} 6^{s}}^{2}}{s_{6} 6^{s}} ;
$$

$$
\hat{s}_{1^{s} 2^{s}}=s_{1^{s} 2^{s}}-\frac{s_{1^{s} 6^{s}} s_{2^{s}} 6^{s}}{s_{6}^{s} 6^{s}},
$$

and where the conventional abbreviated subscripts have been used for the compliance coefficients. Thus, applying the boundary conditions and substituting Eqs. (1) and (3), (4) in Eq. (2), we obtain

$$
\mathrm{e}_{\text {elas }}^{s}=\frac{1}{2}\left(\frac{C_{y} y^{s}}{R_{y}^{2}}+\frac{2 C_{y^{s} z^{s}}}{R_{y} R_{z^{s}}}+\frac{C_{z} z^{s}}{R_{z}^{2}}\right) x^{s 2},
$$

where

$$
C_{y^{s} y^{s}}=\frac{\hat{s}_{2^{s} 2^{s}}}{\hat{s}^{s}}, C_{z^{s} z^{s}}=\frac{\hat{s}_{1^{s} 1^{s}}}{\hat{s}^{s}}, C_{y^{s} z^{s}}=-\frac{\hat{s}_{1^{s} 2^{s}}}{\hat{s}^{s}}
$$

with $\hat{s}^{s}=\hat{s}_{1^{s} 1^{s}} \hat{s}_{2^{s} 2^{s}}-\hat{s}_{1^{s_{2}} 2^{s}}$.

\subsection{Determination of the magnetoelastic stresses from the curvature of the plate}

We should now evaluate the energy density of the plate, i.e., the magnetic film, or SL, plus the substrate. This energy is contributed by: a magnetoelastic one associated to the magnetic sample, which is the origin of the bending of the plate under an applied magnetic field, and by the elastic energies from the substrate and from the magnetic sample.

2.2.1. Magnetoelastic and elastic density energies of the magnetic sample. To first approximation it is assumed that the magnetoelastic hamiltonian terms are quadratic in the spin components and linear in the strains. The magnetoelastic and elastic energy are then written considering only single-ion contributions and taking into account the pointsymmetry group, $4 / m \overline{3} \frac{2}{m}$, for the $\mathrm{REFe}_{2}$ intermetallic compounds, in the form [13]

$$
\begin{aligned}
& \mathrm{e}_{m \mathrm{el+el}}^{s}=b_{0}\left(\varepsilon_{x^{s} x^{s}}+\varepsilon_{y^{s} y^{s}}+\varepsilon_{z^{s} z^{s}}\right)+b_{1}\left(\alpha_{x}^{2} \varepsilon_{x^{s} x^{s}}+\alpha_{y^{s}}^{2} \varepsilon_{y^{s} y^{s}}+\alpha_{z^{s}}^{2} \varepsilon_{z^{s} z^{s}}\right)+b_{2}\left(\alpha_{x^{s}} \alpha_{y^{s}} \varepsilon_{x^{s} y^{s}}+\alpha_{x^{s}} \alpha_{z^{s}} \varepsilon_{x^{s} z^{s}}+\alpha_{z^{s}} \alpha_{y^{s}} \varepsilon_{z^{s} y^{s}}\right)+ \\
& +\frac{1}{2} c_{1^{s} 1^{s}}\left(\varepsilon_{x^{s} x^{s}}^{2}+\varepsilon_{y^{s} y^{s}}^{2}+\varepsilon_{z^{s} z^{s}}^{2}\right)+c_{1^{s} 2^{s}}\left(\varepsilon_{x^{s} x^{s}} \varepsilon_{z^{s} z^{s}}+\varepsilon_{y^{s} y^{s}} \varepsilon_{z^{s s}}+\varepsilon_{x^{s} x^{s}} \varepsilon_{y^{s} y^{s}}\right)+\frac{1}{2} c_{4^{s} 4^{s}}\left(\varepsilon_{x^{s} y^{s}}^{2}+\varepsilon_{x^{s} z^{s}}^{2}+\varepsilon_{z^{s} y^{s}}^{2}\right) .
\end{aligned}
$$

In Eq. (8) the represented energy densities $\mathrm{e}_{m \mathrm{el}}^{\prime}$ and $\mathrm{e}_{m \mathrm{el}}^{\prime \prime}$ are written in the $S^{\prime}$ and $S^{\prime \prime}$ reference systems, respectively; $\alpha_{i}$ denote the direction cosines of the macroscopic magnetisation, $\mathbf{M} ; b_{i}(i=0,1$ and 2$)$ are the magnetoelastic coupling parameters (or magnetoelastic stresses); $\varepsilon$ 's and c's are the cartesian strain and elastic components, respectively, of
$\mathrm{REFe}_{2}$ compounds, for the corresponding reference system.

To evaluate the energy density of the system: substrate-magnetic sample we will assume that the strains in the film or SL are uniform because of it small thickness, compared with the substrate one. This means that, in Eq. (1), the variable $x^{s}$ can be 
substituted by the constant value $\beta h_{\text {subs }}$, the distance between the neutral plane and the bottom of the film or SL, expressed as a fraction $\beta$ of the substrate thickness $h_{\text {subs }}$.

The boundary conditions, extended to the volume of the sample, imply the minimization of its total density energy: $\mathrm{e}_{m \mathrm{el+el}}^{s}$ with respect to $\varepsilon_{x^{s} x^{s}}$, $\varepsilon_{x^{s} y^{s}}, \varepsilon_{x^{s} z^{s}}$ for our two measurement configurations.
So, after that, the Eq. (8) can be rewritten, using Eq. (1), and leave Eq. (8) as a function of the two principal curvatures: $1 / R_{y^{\prime}}$ and $1 / R_{z^{\prime}}$, for $S^{\prime}$, and $1 / R_{y^{\prime \prime}}$ and $1 / R_{z^{\prime \prime}}$, for $S^{\prime \prime}$, and of the position of the neutral plane, $\beta$ (the same for both cases). Thus, the integration of the total energy densities to the corresponding volumes of the substrate and magnetic film, give rise the total energies: for $S^{\prime}$,

$$
E_{m \mathrm{el}}^{\text {tot }}=\left(\alpha^{\prime} \|[010]^{\prime}\right)=A \int_{(\beta-1) h_{\text {subs }}}^{\beta h_{\text {subs }}} \frac{1}{2}\left(\frac{C_{y^{\prime} y^{\prime}}}{R_{y^{\prime}}^{2}}+\frac{2 C_{y^{\prime} z^{\prime}}}{R_{y^{\prime}} R_{z^{\prime}}}+\frac{C_{z^{\prime} z^{\prime}}}{R_{z^{\prime}}^{2}}\right) x^{\prime 2} d x^{\prime}+
$$

$$
\begin{gathered}
+A \int_{\beta h_{\text {subs }}}^{\beta h_{\text {subs }}^{+h}{ }_{m s}}\left\{b_{0}\left(\frac{x^{\prime}}{R_{z^{\prime}}} \frac{c_{11}-c_{12}+2 c_{44}}{c_{11}+c_{12}+2 c_{44}}+\frac{x^{\prime}}{R_{y^{\prime}}} \frac{4 c_{44}}{c_{11}+c_{12}+2 c_{44}}\right)+b_{1}\left(\frac{x^{\prime}}{R_{y^{\prime}}} \frac{c_{12}}{c_{11}+c_{12}+2 c_{44}}\right)+\right. \\
\left.+b_{2}\left(\frac{x^{\prime}}{R_{y^{\prime}}} \frac{c_{11}+c_{12}}{c_{11}+c_{12}+2 c_{44}}+\frac{x^{\prime}}{R_{z^{\prime}}} \frac{c_{12}}{c_{11}+c_{12}+2 c_{44}}\right)\right\} d x^{\prime},
\end{gathered}
$$

and for $S^{\prime \prime}$,

$$
\begin{gathered}
E^{\prime \prime}{ }_{m e l}^{\text {tot }}\left(\alpha^{\prime \prime} \|[010]^{\prime \prime}\right)=A \int_{(\beta-1) h_{\text {subs }}}^{\beta h_{\text {subs }}} \frac{1}{2}\left(\frac{C_{y^{\prime \prime} y^{\prime \prime}}}{R_{y^{\prime \prime}}^{2}}+\frac{2 C_{y^{\prime \prime} z^{\prime \prime}}}{R_{y^{\prime \prime}} R_{z^{\prime \prime}}}+\frac{C_{z^{\prime \prime} z^{\prime \prime}}}{R_{z^{\prime \prime}}^{2}}\right) x^{\prime \prime 2} d x^{\prime \prime}+ \\
+A \int_{\beta h_{\text {subs }}}^{\beta h_{\text {subs }} h_{m s}}\left\{-\frac{b_{0}}{3}\left(\frac{x^{\prime \prime}}{R_{y^{\prime \prime}}} \frac{c_{12}-c_{11}-10 c_{44}}{c_{11}+c_{12}+2 c_{44}}-\frac{x^{\prime \prime}}{R_{z^{\prime \prime}}} \frac{c_{12}-c_{11}-4 c_{44}}{c_{11}+c_{12}+2 c_{44}}\right)-\frac{b_{1}}{9}\left(\frac{x^{\prime \prime}}{R_{y^{\prime \prime}}} \frac{c_{12}-c_{11}-10 c_{44}}{c_{11}+c_{12}+2 c_{44}}+\right.\right. \\
\left.\left.+2 \frac{x^{\prime \prime}}{R_{z^{\prime \prime}}} \frac{c_{12}-c_{11}-4 c_{44}}{c_{11}+c_{12}+2 c_{44}}\right)+\frac{2 b_{2}}{9}\left(\frac{x^{\prime \prime}}{R_{y^{\prime \prime}}} \frac{4 c_{11}+5 c_{12}+4 c_{44}}{c_{11}+c_{12}+2 c_{44}}+\frac{x^{\prime \prime}}{R_{z^{\prime \prime}}} \frac{c_{12}-c_{11}-4 c_{44}}{c_{11}+c_{12}+2 c_{44}}\right)\right\} d x^{\prime \prime},
\end{gathered}
$$

where $A$ is the area of the plate.

2.2.2. Relation between curvatures and magnetoelastic stresses parameters. For the magnetic sample, we have assumed it is uniformly strained, so: $\varepsilon_{y^{\prime} y^{\prime}}=\beta h_{\text {subs }} / R_{y^{\prime}}$ and $\varepsilon_{z^{\prime} z^{\prime}}=\beta h_{\text {subs }} / R_{z^{\prime}}$ for $S^{\prime}$, and $\varepsilon_{y^{\prime \prime} y^{\prime \prime}}=\beta h_{\text {subs }} / R_{y^{\prime \prime}}$ and $\varepsilon_{z^{\prime \prime} z^{\prime \prime}}=\beta h_{\text {subs }} R_{z^{\prime \prime}}$ for $S^{\prime \prime}$ performing the integration operation of Eqs. (9) and (10), we obtain: for $S^{\prime}$,

$$
E_{m \mathrm{el}}^{\mathrm{tot}}\left(\alpha^{\prime} \|[010]^{\prime}\right)=A h_{\mathrm{subs}}^{3}\left(\beta^{2}-\beta+\frac{1}{3}\right) \frac{1}{2}\left(\frac{C_{y^{\prime} y^{\prime}}}{R_{y^{\prime}}^{2}}+\frac{2 C_{y^{\prime} z^{\prime}}}{R_{y^{\prime}} R_{z^{\prime}}}+\frac{C_{z^{\prime} z^{\prime}}}{R_{z^{\prime}}^{2}}\right)+A h_{m s}\left\{b _ { 0 } \left(\frac{\beta h_{\mathrm{subs}}}{R_{z^{\prime}}} \frac{c_{11}-c_{12}+2 c_{44}}{c_{11}+c_{12}+2 c_{44}}+\right.\right.
$$


$\left.+\frac{\beta h_{\text {subs }}}{R_{y^{\prime}}} \frac{4 c_{44}}{c_{11}+c_{12}+2 c_{44}}\right)+b_{1}\left(\frac{\beta h_{\text {subs }}}{R_{y^{\prime}}} \frac{c_{12}}{c_{11}+c_{12}+2 c_{44}}\right)+b_{2}\left(\frac{\beta h_{\text {subs }}}{R_{y^{\prime}}} \frac{c_{11}+c_{12}}{c_{11}+c_{12}+2 c_{44}}+\frac{\beta h_{\text {subs }}}{R_{z^{\prime}}} \frac{c_{12}}{c_{11}+c_{12}+2 c_{44}}\right)$,

and for $S^{\prime \prime}$,

$$
\begin{gathered}
E_{m \mathrm{el}}^{\prime \text { tot }}\left(\alpha^{\prime \prime} \|[010]^{\prime \prime}\right)=A h_{\text {subs }}^{3}\left(\beta^{2}-\beta+\frac{1}{3}\right) \frac{1}{2}\left(\frac{C_{y^{\prime \prime} y^{\prime \prime}}}{R_{y^{\prime \prime}}^{2}}+\frac{2 C_{y^{\prime \prime} z^{\prime \prime}}}{R_{y^{\prime \prime}} R_{z^{\prime \prime}}}+\frac{C_{z^{\prime \prime} z^{\prime \prime}}}{R_{z^{\prime \prime}}^{2}}\right)+A h_{m s}\left\{-\frac{b_{0}}{3}\left(\frac{\beta h_{\text {subs }} v c_{12}-c_{11}-10 c_{44}}{R_{y^{\prime \prime}}} \frac{c_{11}+c_{12}+2 c_{44}}{c_{44}}\right.\right. \\
\left.-\frac{\beta h_{\text {subs }}}{R_{z^{\prime \prime}}} \frac{c_{12}-c_{11}-4 c_{44}}{c_{11}+c_{12}+2 c_{44}}\right)-\frac{b_{1}}{9}\left(\frac{\beta h_{\text {subs }}}{R_{y^{\prime \prime}}} \frac{c_{12}-c_{11}-10 c_{44}}{c_{11}+c_{12}+2 c_{44}}+2 \frac{\beta h_{\text {subs }}}{R_{z^{\prime \prime}}} \frac{c_{12}-c_{11}-4 c_{44}}{c_{11}+c_{12}+2 c_{44}}\right)+ \\
\left.+\frac{2 b_{2}}{9}\left(\frac{\beta h_{\text {subs }}}{R_{y^{\prime \prime}}} \frac{4 c_{11}+5 c_{12}+4 c_{44}}{c_{11}+c_{12}+2 c_{44}}+\frac{\beta h_{\text {subs }}}{R_{z^{\prime \prime}}} \frac{c_{12}-c_{11}-4 c_{44}}{c_{11}+c_{12}+2 c_{44}}\right)\right\}
\end{gathered}
$$

In this situation, the minimization of the energies given in Eqs. (11) and (12) with respect to the four independent parameters: $\beta, R_{y^{\prime \prime}}^{-1}$ and $R_{z^{\prime \prime}}^{-1}$ for $S^{\prime}$ and $R_{z^{\prime \prime}}^{-1}$ for $S^{\prime \prime}$, leads to $\beta=2 / 3$ and to the following expressions:

$$
\begin{gathered}
\frac{\partial E_{\text {tot }}^{\prime}(\alpha \|[\overline{1} 10])}{\partial R_{y^{\prime}}^{-1}}=\tilde{\sigma}([\overline{1} 10],[\overline{1} 10])+ \\
+\frac{2}{3} \frac{b_{2}\left(c_{11}+c_{12}\right)+2 c_{44}\left(b_{1}+2 b_{0}\right)}{c_{11}+c_{12}+2 c_{44}}=0 \\
\frac{\partial E_{\text {tot }}^{\prime}(\alpha \|[\overline{1} 10])}{\partial R_{z^{\prime}}^{-1}}=\tilde{\sigma}([\overline{1} 10],[001])+ \\
+\frac{\left(b_{2}-b_{1}\right) c_{12}+b_{0}\left(c_{11}-c_{12}+2 c_{44}\right)}{c_{11}+c_{12}+2 c_{44}}=0 \\
\frac{\partial E^{\prime \prime}{ }_{\text {tot }}(\alpha \|[\overline{1} 11])}{\partial R_{z^{\prime \prime}}^{-1}}=\tilde{\sigma}([\overline{1} 11],[1 \overline{1} 2])- \\
-\frac{2}{9}\left(b_{1}-b_{2}+2 b_{0}\right)\left[\frac{-c_{11}+c_{12}-4 c_{44}}{c_{11}+c_{12}+2 c_{44}}\right]=0
\end{gathered}
$$

where we have defined the $\tilde{\sigma}(\alpha, \beta)$ MEL stresses as follows,

$$
\tilde{\sigma}([\overline{1} 10],[\overline{1} 10]) \equiv \frac{1}{6} \frac{h_{\mathrm{subs}}^{2}}{h_{m s}}\left(\frac{C_{y^{\prime} y^{\prime}}}{R_{y^{\prime}}}+\frac{C_{z^{\prime} y^{\prime}}}{R_{z^{\prime}}}\right)
$$

$$
\begin{aligned}
& \tilde{\sigma}([\overline{1} 10],[001]) \equiv \frac{1}{6} \frac{h_{\text {subs }}^{2}}{h_{m s}}\left(\frac{C_{z^{\prime \prime} z^{\prime \prime}}}{R_{z^{\prime \prime}}}+\frac{C_{y^{\prime \prime} z^{\prime \prime}}}{R_{y^{\prime \prime}}}\right), \\
& \tilde{\sigma}([\overline{111}], 1 \overline{1} 2]) \equiv \frac{1}{6} \frac{h_{\text {subs }}^{2}}{h_{m s}}\left(\frac{C_{z^{\prime \prime} z^{\prime \prime}}}{R_{z^{\prime \prime}}}+\frac{C_{y^{\prime \prime} z^{\prime \prime}}}{R_{y^{\prime \prime}}}\right) .
\end{aligned}
$$

Notice that we have minimized the total energies for the three different cases to obtain the minimum number of equations needed to obtain the three relevant MEL stresses parameters, $b_{0}, b_{1}$, and $b_{2}$. By solving the system of Eqs. (13), (14), and (15) we obtain these MEL parameters as a function of the MEL stresses which can be determined from the experimental results (see Sec. 3 below). In this way just with an unique magnetostrictive experiment, it has been possible to determine all the second order MEL contributions to the total MEL energy, which, in fact, is the most relevant one.

Let us compare the above expressions with the obtained ones for the hexagonal case, when the magnetization of the sample is parallel to the $x$ axis $\left(\alpha_{x}=1, \alpha_{y}=\alpha_{z}=0\right)$. In this situation, the minimization of the total energy of the system with respect to the three independent parameters, $\beta, 1 / R_{x}$ and $1 / R_{y}$, leads to the expressions

$\frac{1}{9} A h_{\text {subs }}^{3}\left(\frac{C_{x x}}{R_{x}}+\frac{C_{x y}}{R_{y}}\right)-\frac{2}{3} A h_{\text {subs }} h_{m s}\left(\mathcal{B}+\frac{1}{4} B^{\gamma, 2}\right)=0$ 
$\frac{1}{9} A h_{\text {subs }}^{3}\left(\frac{C_{x y}}{R_{x}}+\frac{C_{y y}}{R_{y}}\right)-\frac{2}{3} A h_{\text {subs }} h_{m s}\left(\mathcal{B}-\frac{1}{4} B^{\gamma, 2}\right)=0$,

and $\beta=2 / 3$, which has been already substituted in Eqs. (19) and (20). In these equations $\mathcal{B}$ represents a complicated combination of symmetry elastic constants, $c_{j k}^{\mu}$, and magnetoelastic stresses, $B_{p}^{\mu l}$, for the magnetic sample, which reads

$$
\begin{aligned}
\mathcal{B} & =-\frac{1}{2}\left\{\left(B_{1}^{\alpha, 0}-\frac{1}{3} B_{1}^{\alpha, 2}\right) \frac{\sqrt{3} c_{12}^{\alpha}+c_{22}^{\alpha}}{c_{11}^{\alpha}+2 / \sqrt{3} c_{12}^{\alpha}+1 / 3 c_{22}^{\alpha}}-\right. \\
& \left.-\left(B_{2}^{\alpha, 0}-\frac{1}{3} B_{2}^{\alpha, 2}\right) \frac{\sqrt{3} c_{11}^{\alpha}+c_{12}^{\alpha}}{c_{11}^{\alpha}+2 / \sqrt{3} c_{12}^{\alpha}+1 / 3 c_{22}^{\alpha}}\right\} .
\end{aligned}
$$

Note that the volume magnetoelastic constants appearing in Eq. (21) include the exchange contribution to the volume strain.

We have obtained the expressions (13), (14), and (15) and (19) and (20), which relate the principal curvatures of a free plate with the magnetoelastic stresses originated upon the application of a magnetic field. However, this is not the real situation in our experiments. In the next paragraph we explain the actual situation as well as details about the experimental technique and characteristics of the measured samples.

\section{Experimental technique and samples}

\subsection{Experimental arrangement}

Our samples, which are rectangular (typical plane dimensions of about $10 \mathrm{~mm} \times 10 \mathrm{~mm})$, are clamped along one of their edges (cantilever configuration) (Fig. 1). The exact analytical solution of this kind of problem is unfeasible, because the boundary conditions can not be properly imposed. To take into account the effect of the clamping on the deflection of the plate, finite element modeling [24] and approximate analytical solutions [25] have been proposed. However, we cannot apply the results obtained in these approaches to the problem, which are based on the determination of the deflection of the free end of the clamped plate. In our experimental set-up, we use a capacitive technique which allows us to measure the change of capacitance related with the overall curvatures of the plate. Since the equation representing the plate's surface cannot be known, we associate this change

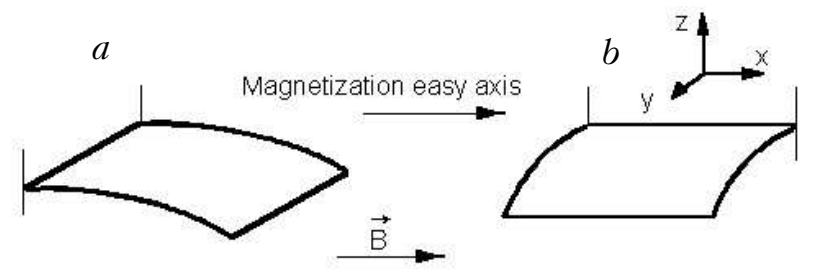

Fig. 1. Experimental arrangement of the cantilever: bending along the direction of the applied magnetic field $(a)$; bending perpendicular to the direction of the field $(b)$.

of capacitance to a single curvature of the plate. Our hypothesis is that the curvature of every line parallel to the clamping line is much smaller than the curvature which appears perpendicularly to the clamping direction. We will neglect such small curvatures not only close to the clamping line but also for the whole plate. This approximation overestimates the value of the deflection and, hence, of the magnetoelastic parameters deduced from it, which can have an error of order 5\% [26] for our approximately squared samples.

Thus, in the case of cylindrical symmetry, for the sample clamped along the $y$ direction (Fig. 1,a) we will only consider Eq. (19), with $R_{y}^{-1}=0$, i.e.,

$\frac{\partial E_{\text {tot }}}{\partial R_{x}^{-1}}=\frac{1}{9} A h_{\text {subs }}^{3} \frac{C_{x x}}{R_{x}}-\frac{2}{3} A h_{\text {subs }} h_{m s}\left(\mathcal{B}+\frac{1}{4} B^{\gamma, 2}\right)=0$,

while for experiments with the sample clamped along $x$, we will use Eq. (20), with $R_{x}^{-1}=0$, which reads

$\frac{\partial E_{\text {tot }}}{\partial R_{y}^{-1}}=\frac{1}{9} A h_{\text {subs }}^{3} \frac{C_{y y}}{R_{y}}-\frac{2}{3} A h_{\text {subs }} h_{m s}\left(\mathcal{B}-\frac{1}{4} B^{\gamma, 2}\right)=0$.

From Eqs. (22) and (23) it is practical to define the magnetoelastic stresses acting along the $O X$ and $O Y$ directions,

$$
\tilde{\sigma}(x, x)=\mathcal{B}+\frac{1}{4} B^{\gamma, 2}, \tilde{\sigma}(x, y)=\mathcal{B}-\frac{1}{4} B^{\gamma, 2},
$$

where the first letter within the brackets stands for the direction of the applied magnetic field and, the second one, for the direction along which the plate deforms longitudinally under the effect of the magnetoelastic stresses. Subtraction of the above stresses allows us to obtain the magnetoelastic stress parameter $B^{\gamma, 2}$. Thus, we get 


$$
B^{\gamma, 2}=2[\tilde{\sigma}(x, x)-\tilde{\sigma}(x, y)]=\frac{1}{3} \frac{h_{\text {subs }}^{2}}{h_{m s}}\left(\frac{C_{x x}}{R_{x}}-\frac{C_{y y}}{R_{y}}\right) .
$$

Therefore, by performing two curvature measurements, according to the two different configurations shown in Fig. 1, we can separate the parameter $B^{\gamma, 2}$, associated with the magnetoelastic strain $\varepsilon_{1}^{\gamma}=1 / 2\left(\varepsilon_{x x}-\varepsilon_{y y}\right)$ within the $(\mathbf{a}, \mathbf{b})$ basal plane of the hcp structure of the magnetic sample. The subtraction eliminates, not only the volume strain, $\varepsilon_{1}^{\alpha}$, and tetragonal strain, $\varepsilon_{2}^{\alpha}$, terms but also the effect of the differential thermal expansion between magnetic sample and substrate in the zero-field capacitance. Note that both kind of measurements should be performed with the field applied along the magnetic easy direction of the sample, i.e., the $\mathbf{a}$ or $\mathbf{b}$ axes of the basal plane for rare-earth-based samples, provided that their growing plane is the basal plane.

In the case of cubic symmetry we should perform at least three curvature measurements to be able to determine the three relevant MEL stress parameters, as we described in the previous section. Expressions similar to Eq. (25), in which the only elastic constants appearing are those of the substrate, are not obtained now. Instead of it, by solving the system of Eqs. (13), (14), and (15), we get for $b_{0}, b_{1}$ and $b_{2}$ relationships where the radii of curvature are multiplied by complex combinations of all the elastic constants, for the substrate and the magnetic sample.

\subsection{Cantilever capacitive technique}

The determination of the curvature of the plate is performed by means a capacitive cell, in which the metallic magnetic sample deposited on the substrate acts as one of the electrodes. The rest of the cell was made in copper, and annealed at $800 \mathrm{~K}$ to improve its behavior under thermal cycling. Measurements where done either in a three terminal capacitor configuration with a AEL Ltd. ratio bridge (sensitivity: $10^{-6} \mathrm{pF}$ ), or in a two plates capacitor configuration with an Andeen-Hagerling 2500 A capacitance bridge (sensitivity: $5 \cdot 10^{-7} \mathrm{pF}$ ). No differences were found between both methods, except for a small difference in the signal to noise ratio in favour of the latter case. In this situation, cantilever deflections as small as $10^{-9} \mathrm{~m}$ can be detected for a typical sample length of $10 \mathrm{~mm}$.

For small deflections, it is straightforward to show that a curvature $R^{-1}$ of the cantilever produces a capacitance change given by the expression

$$
\Delta C=-C_{0}^{2} L^{2} / 6 \varepsilon_{0} A R,
$$

where $C_{0}$ is the capacitance of the parallel plate capacitor, $A$ and $L$ respectively, are the area and length of the plate; $\varepsilon_{0}$ is the permittivity of vacuum. For instance, for hexagonal systems, expression (25), together with Eq. (26), gives the magnetoelastic stress $B^{\gamma, 2}$ in terms of the different experimental values for both kind of measurements, i.e., sample clamped along $y$ and $x$ directions, in the form

$$
\begin{gathered}
B^{\gamma, 2}=-\frac{2 \varepsilon_{0} h_{\text {subs }}^{2}}{h_{m s}} \times \\
\times\left(C_{x x} \frac{A_{(x, x)} \Delta C_{(x, x)}}{L_{(x, x)}^{2} C_{0(x, x)}^{2}}-C_{y y} \frac{A_{(x, y)} \Delta C_{(x, y)}}{L_{(x, y)}^{2} C_{0(x, y)}^{2}}\right),
\end{gathered}
$$

where $C_{x x}$ and $C_{y y}$ are given by expressions similar to those of Eq. (7) (see Ref. 6), with the compliance coefficients corresponding to the substrate material, and the labels $(x, x)$ and $(x, y)$ refer to the two measuring configurations.

In the experimental setup the capacitive cell is placed within a continuous flow cryostat, allowing us to measure between 1.7 and $300 \mathrm{~K}$. Magnetic fields up to $12 \mathrm{~T}$, produced by a superconducting coil, can be applied parallel to the plane of the sample.

\subsection{Samples characteristics}

The magnetoelastic stress experiments reported in this paper were performed in Ho films and in $\mathrm{Ho} / \mathrm{Y}$, Ho / Lu, Dy / Y, Er / Lu, and Ho/Tm superlattices and in different $\mathrm{REFe}_{2}$ films and bilayers.

All the RE superlattices were grown by molecular beam epitaxy using a Balzers UMS 630 facility. The rare-earth metals grow epitaxially onto a $\mathrm{Nb}$ metal layer deposited on a sapphire substrate [3]. Both the body-centered-cubic $\mathrm{Nb}$ and hexagonalclose-packed rare-earth metals grow with their respective close-packed atomic planes parallel to the substrate plane. The epitaxial relationships are $\{11 \overline{2} 0\} \mathrm{Al}_{2} \mathrm{O}_{3}\|\{110\} \mathrm{Nb}\|\{0001\} \mathrm{RE}$, resulting the $a$ axis of the rare earth at an angle of $5^{\circ}$ with [0001] $\mathrm{Al}_{2} \mathrm{O}_{3}$. The crystalline structure of the superlattices was investigated using a triple-axis x-ray diffractometer, giving an interface width of \pm 2 lattice planes [3]. The sapphire substrates, of initial thickness of $500 \mu \mathrm{m}$, were thinned down to $150 \mu \mathrm{m}$ to increase the sensitivity of the cantilever method (note the high value of the elastic constants of sapphire, $\sim 400 \mathrm{GPa}$ ). After the thinning, the mag- 
netic-sample thickness to substrate thickness ratio remains very small $\left(\sim 10^{-3}\right)$. This allows us to disregard the terms of type $\left(R_{i} R_{j}\right)^{-1}$, appearing in $E_{m e l}^{\text {tot }}$, which arise from the elastic energy of the magnetic sample and which are $h_{m a} / h_{\text {subs }}$ times smaller than the corresponding ones in the elastic energy of the substrate.

From x-ray diffraction measurements performed in our $\mathrm{Ho} / \mathrm{Y}$ and $\mathrm{Ho} / \mathrm{Lu}$ superlattices, between $\mathrm{RT}$ and $10 \mathrm{~K}$ [28], we know that the epitaxy is good: the misfit between basal plane lattice parameters of $\mathrm{Ho}$ and $\mathrm{Lu}$ (or Y) in the superlattice is very small (e.g., for the hexagonal a lattice parameter: $\left(a^{\mathrm{Y}}-a^{\mathrm{Ho}}\right) / a^{\mathrm{Ho}}=2 \cdot 1 \cdot 10^{-3}$, at $45 \mathrm{~K}$ in a $\left.\left[\mathrm{Ho}_{40} / \mathrm{Y}_{15}\right]_{50} \mathrm{SL}\right)$.

The $\mathrm{REFe}_{2}$ films studied in this work have been also grown by MBE. Sapphire substrates were covered with a niobium buffer (the epitaxial relationships being: [1 $1 \overline{1} 1] \mathrm{Nb} \|[0001] \mathrm{Al}_{2} \mathrm{O}_{3}$ and [11̄] $\mathrm{Nb} \|[10 \overline{1} 0] \mathrm{Al}_{2} \mathrm{O}_{3}$ ). After that, a very thin iron layer of $15 \AA$ thick was deposited at $820 \mathrm{~K}$ onto the (110) niobium plane reacting with it to produce a NbFe- $\varphi$ alloy on the surface [29]. RHEED surface analyses have shown a $2 D$ rectangular lattice, where the lattice parameters were close to those of a $C 15$ cubic Laves phase in (110) plane, $7.0 \pm 0.1 \AA$ and $4.8 \pm 0.1 \AA$. The $\mathrm{TbFe}_{2}$ layers were obtained by co-deposition of the rare-earth and iron constituents, keeping the substrate at $820 \mathrm{~K}$ for $\mathrm{YFe}_{2}$ layer deposition, and reducing the temperature to $620 \mathrm{~K}$ for $\mathrm{TbFe}_{2}$ layer in order to avoid interdiffusion. At this level the epitaxial relationships are: [11̄0] $\mathrm{REFe}_{2} \|[1 \overline{1} 0] \mathrm{Nb}$ and [001] $\mathrm{REFe}_{2} \|[001] \mathrm{Nb}$. Finally, all the samples were overcoated with a $200 \AA$ thick layer of Y to protect them from oxidation. A small average roughness $(\approx 25 \AA)$ of the layers was confirmed by Atomic Force Microscopy [30] in all the samples. However $\mathrm{TbFe}_{2}(300 \AA) / \mathrm{YFe}_{2}(1000 \AA)$ sample presented islands quite close each other, in fact quasi-continuously spread on the plane, and elongated along [110] direction. The composition of the bilayers was checked by microprobe analysis. The stoichiometric composition was found to be within $\pm 2 \%$ for $\mathrm{REFe}_{2}(\mathrm{RE}=\mathrm{Tb}$ and $\mathrm{Y})$. The thickness was estimated by the calibration of the evaporation rates, using quartz balances and optical sensors with a $10 \%$ of error; x-ray diffraction scattering confirmed that all the films grown epitaxially on the NbFe- $\varphi$ buffer. They exhibited an average $\approx 0.6 \%$ lattice expansion along [110]. The mismatch lattice parameter between $\mathrm{TbFe}_{2}$ and $\mathrm{YFe}_{2}$ layers induces a shear strain at the interface, $\varepsilon_{x y}^{0} \approx-0.61,-0.58$, -0.57 , and $-0.54 \%$ for $\mathrm{TbFe}_{2}$ samples thickness of
300, 600, 1000, and $1300 \AA$, respectively [30,31]. The dispersion observed along the [220] orientation corresponds to an average mosaic spread of $\simeq 1.5^{\circ}$, while the Bragg peaks broadening along this orientation also indicates a mean coherence length ranged between 200 and $400 \AA$. In addition, analyses of Mössbauer spectra carried out on the samples indicate that [1111] is the magnetization easy direction in absence of applied magnetic field at room temperature [30]. However, a careful analysis of these results has also concluded that the magnetization easy axis would be strongly influenced by the shear deformation existing in the growing plane, which is temperature dependent [30].

\section{Magnetoelasticity of Ho films}

We have measured the magnetoelastic stress in two epitaxial Ho films, 5000 and $10000 \AA$ thick [32]. The interest in these measurements is double: first, they are useful for checking the experimental system and the validity of the hypotheses assumed in the analysis explained in Sec. 2, as well as the clamping conditions; second, the results obtained in Ho films can be a reference for magnetoelastic stress measurements in Ho-based superlattices, although the studied samples, being so thick, are expected to
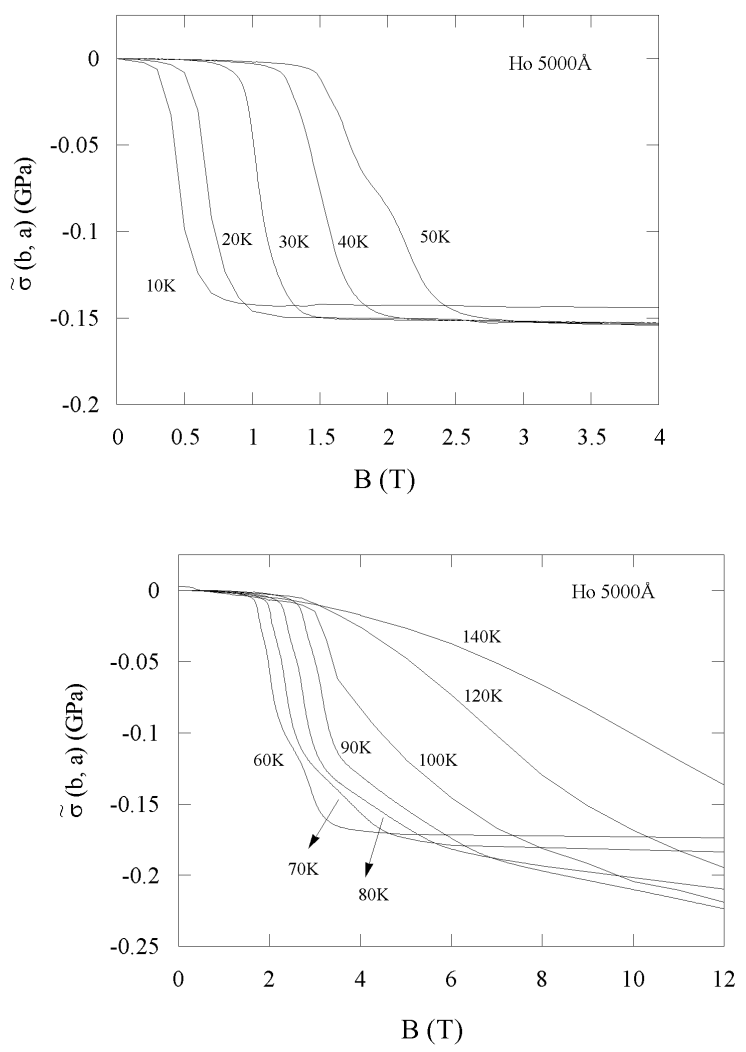

Fig. 2. Magnetoelastic stress isotherms $\tilde{\sigma}(b, a)$ for the 5000 A Ho film. 


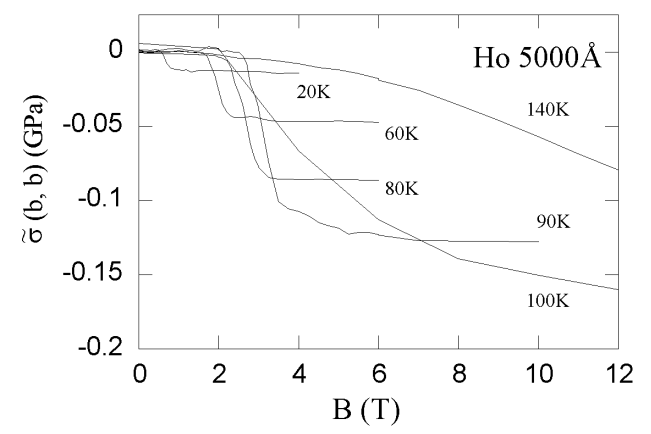

Fig. 3. Magnetoelastic stress isotherms $\tilde{\sigma}(b, b)$ for the $5000 \AA$ Ho film.

exhibit a bulk-like behavior in their magnetic and magnetoelastic properties.

In Figs. 2 and 3 we show the magnetoelastic stress isotherms obtained for the $5000 \AA$ Ho film between 10 and $140 \mathrm{~K}$. The measurements were performed applying the magnetic field $\mathbf{B}$ along the b-easy direction of holmium and with the sample clamped along the $b$ axis, $\tilde{\sigma}(b, a)$ in Fig. 2, and along the $a$ axis, $\tilde{\sigma}(b, b)$ in Fig. 3.

The magnetoelastic stress isotherms display changes in the slope at certain critical fields that can be ascribed to field induced transitions to different magnetic phases. However, the study of the phase diagrams and the analysis of the differences observed in the critical fields when compared with the bulk Ho values (in the films, the critical fields are higher [33], are not aims of the present work. We will only stress that, at the maximum applied field of $12 \mathrm{~T}$, for temperatures below $\sim 90 \mathrm{~K}$, we have reached a ferromagnetic phase and we can safely assure that the sample is saturated along the easy axis.

Subtraction of the values of both magnetoelastic stresses (see Eq. (25)), for the different temperatures and at the maximum field of $12 \mathrm{~T}$, gives the temperature variation of the magnetoelastic stress parameter $B^{\gamma, 2}$, which is plotted in Fig. 4 for the $5000 \AA$ film. We show in the same figure the fit of the experimental results by using the expression given by the standard Callen and Callen theory for the magnetoelastic coupling [27], which reads

$$
B^{\gamma, 2}(T)=B^{\gamma, 2}(0) \hat{I}_{5 / 2}\left[\mathcal{L}^{-1}(m(T))\right],
$$

where $m$ is the reduced magnetization of the Ho film $(m=M(T) / M(0)) ; \hat{I}_{5 / 2}$ is the reduced hyperbolic Bessel function, $I_{l+1 / 2} / I_{1 / 2}$, of order $l=2$; $\mathcal{L}^{-1}$ is the inverse Langevin function. The $0 \mathrm{~K}$ value of the $B^{\gamma, 2}$ stress obtained from the fit was $B^{\gamma, 2}(0)=0.29 \pm 0.02 \mathrm{GPa}$. Similar results were ob-

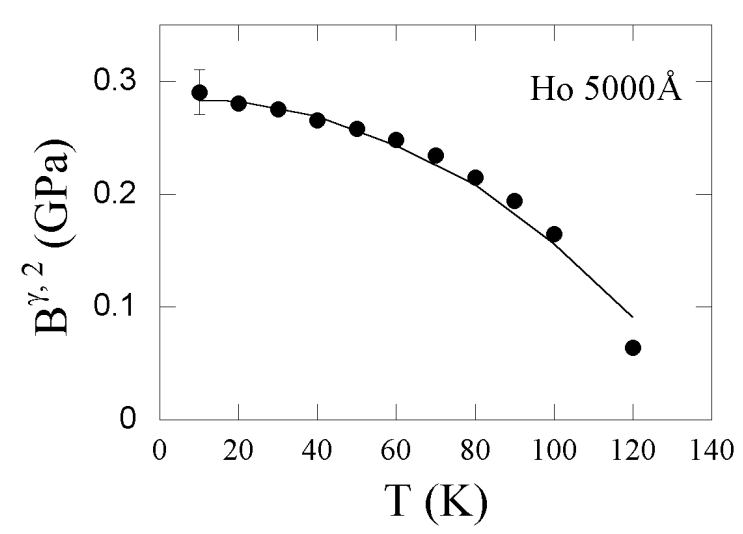

Fig. 4. Thermal variation of the basal plane magnetoelastic stress parameter for the $5000 \AA$ Ho film. The continuous line is the fit to $B^{\gamma, 2}(0) \hat{I}_{5 / 2}\left[\mathcal{L}^{-1}(m(T))\right]$, with $B^{\gamma, 2}(0)=0.29 \mathrm{GPa}$.

tained for the $10000 \AA$ film, for which $B^{\gamma, 2}(0)=$ $0.28 \pm 0.02 \mathrm{GPa}$. Both values are in good agreement with the basal-plane-symmetry-breaking magnetoelastic stress parameter determined for bulk Ho, in which an extrapolation from $B=3 \mathrm{~T}$ and $T=70 \mathrm{~K}$ gives $B^{\gamma, 2}(0)=0.275 \mathrm{GPa}[34]$.

From the above results, i.e., the thermal dependence and the values of the magnetoelastic stress $B^{\gamma, 2}$, we conclude that the origin of the magnetoelastic strain in our Ho films is a single-ion crystal electric field interaction. In addition, as anticipated, this strain is not affected by any surface effect, likely due to the present high volume to surface ratio. Then, in this case, $B^{\gamma, 2} \equiv B_{v}^{\gamma, 2}$. Let us label this volume stress parameter obtained for bulk-like films as $B_{v 0}^{\gamma, 2}$.

\section{Magnetoelastic behavior of $\mathrm{Ho} / \mathrm{Lu}$ superlattices}

Magnetization and MEL stress measurements have been performed in a series of $\left[\mathrm{Ho}_{n_{\mathrm{Ho}}} / \mathrm{Lu}_{n_{\mathrm{Lu}}}\right]_{50}$ superlattices, with $n_{\mathrm{Ho}}$ ranging from 8 to 85 atomic planes. Here we only report on the stress measurements in the $\mathrm{Ho}_{n_{\mathrm{Ho}}} / \mathrm{Lu}_{15}$ samples, although the magnetization isotherms and isofields also provide valuable information about the different field-induced and zero-field magnetic phase transitions [33]. In this regard, the most remarkable fact is the effect of the compressive strain in the basal plane of Ho layers due to the $\mathrm{Lu}$ interleaving layers; this compression, and the corresponding $c$-axis expansion, stabilizes the ferromagnetic phase $[3,8]$. Moreover, the ferromagnetic transition temperature increases for decreasing Ho fraction and the helifan phases, intermediary between the helix and fan phases, existing in bulk Ho [35] and also observed by us in the Ho films, do not appear in the Ho/ Lu 


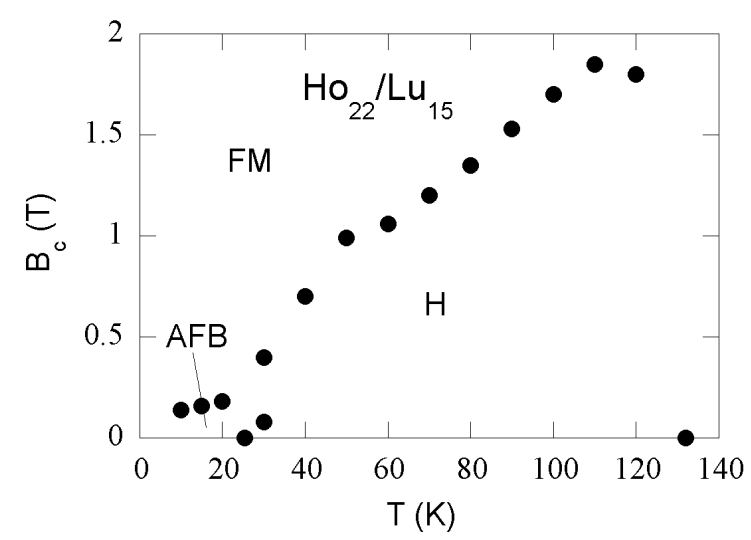

Fig. 5. Magnetic phase diagram for the $\mathrm{Ho}_{22} / \mathrm{Lu}_{15}$ superlattice. The critical fields $B_{c}$ were obtained from magnetoelastic stress and magnetization measurements; FM and $\mathrm{H}$ correspond to the ferromagnetic and helix phases; AFB is a phase where FM Ho blocks are antiferromagnetically coupled [8].

superlattices. In the $\mathrm{Ho} / \mathrm{Lu}$ superlattices with low number of atomic planes of Ho, even the fan phase is absent [33] (Fig. 5).

In Fig. 6 we display, as an example, the $\tilde{\sigma}(b, a)$ and $\tilde{\sigma}(b, b)$ magnetoelastic stresses for the thin Ho layers superlattice $\mathrm{Ho}_{8} / \mathrm{Lu}_{15}$. The isotherms were done in the temperature range from 10 to $160 \mathrm{~K}$ (the lower limit of $10 \mathrm{~K}$ was chosen to avoid the


Fig. 6. Magnetoelastic stress isotherms for the $\mathrm{Ho}_{8} / \mathrm{Lu}_{15}$ superlattice. superconductivity of the $\mathrm{Nb}$ layer present in the samples, appearing below $9 \mathrm{~K}$, which uncontrollably modifies the magnetization and the magnetoelastic stress measurements). The magnetoelastic stress isotherms show anomalies similar to those displayed by the magnetization isotherms. We ascribe the abrupt jumps at the lowest temperatures to transitions to a ferromagnetic state (e.g., see in Fig. 6 the $\tilde{\sigma}(b, a)$ isotherms, at $T \leq 40)$. Smoother anomalies at higher temperatures, which are better detected in the field derivative of the stress isotherms, correspond to helix-fan or helix-ferromagnet transitions. In the paramagnetic phase, $T>T_{N}$, we observe the usual $B^{2}$ thermodynamic behavior.

The basal plane magnetoelastic stress parameter $B^{\gamma, 2}$, which appears in the magnetoelastic energy [6], is determined by using Eq. (25). However, there is a difference with the case of the Ho films: for a superlattice $\left[\mathrm{A}_{t_{A}} / \mathrm{B}_{t_{B}}\right]_{r}$ which has only a volume fraction $F_{\mathrm{A}}=t_{\mathrm{A}} /\left(t_{\mathrm{A}}+t_{\mathrm{B}}\right)$ of material $\mathrm{A}$ undergoing magnetoelastic strain, the value that we actually obtain from the experiment is $B_{\text {meas }}^{\gamma, 2}=$ $=B^{\gamma, 2} f_{\mathrm{A}}$. In our Ho/Lu superlattices we consider that only the Ho layers behave magnetoelastically. Also, because the c-axis parameters of both Ho and $\mathrm{Lu}$ are very similar, the ratio between thicknesses $f_{\mathrm{A}}$ can be well approximated by the ratio between numbers of atomic layers. Therefore, for $\mathrm{A}=\mathrm{Ho}$ and $B=\mathrm{Lu}$, we have

$$
B_{\text {meas }}^{\gamma, 2}=B^{\gamma, 2} \frac{n_{\mathrm{Ho}}}{n_{\mathrm{Ho}}+n_{\mathrm{Lu}}} .
$$

For convenience, as we will see below, we have plotted in Fig. 7 the values, at $10 \mathrm{~K}$ and $12 \mathrm{~T}$, of $B_{\text {meas }}^{\gamma, 2}\left(n_{\mathrm{Ho}}+n_{\mathrm{Lu}}\right)$ (with $\left.n_{\mathrm{Lu}}=15\right)$ as a function of the number of Ho atomic planes in the bilayer repeat, $n_{\mathrm{Ho}}$. In the next section we will see that the observed non-linear dependence of the product $B_{\text {meas }}^{\gamma, 2}\left(n_{\mathrm{Ho}}+n_{\mathrm{Lu}}\right)$ with $n_{\mathrm{Ho}}$ denotes the presence of additional terms in the magnetoelastic stress, i.e., in the present SL's $B^{\gamma, 2}$ is not simply $B_{v 0}^{\gamma, 2}$ (the volume magnetoelastic stress obtained for the Ho films). If the latter case would be true we would obtain the dashed line shown in Fig. 7, as it is obvious from Eq. (29).

\subsection{Analysis of the basal plane cylindrical symmetry breaking magnetoelastic stress}

In a previous work [4], to explain the anomalous thermal dependence of the basal plane magnetoelastic stress in a $\left[\mathrm{Ho}_{6} / \mathrm{Y}_{6}\right]_{100}$ superlattice, we showed that it was necessary to consider that the associated magnetoelastic parameter $B^{\gamma, 2}$ had a linear depend- 

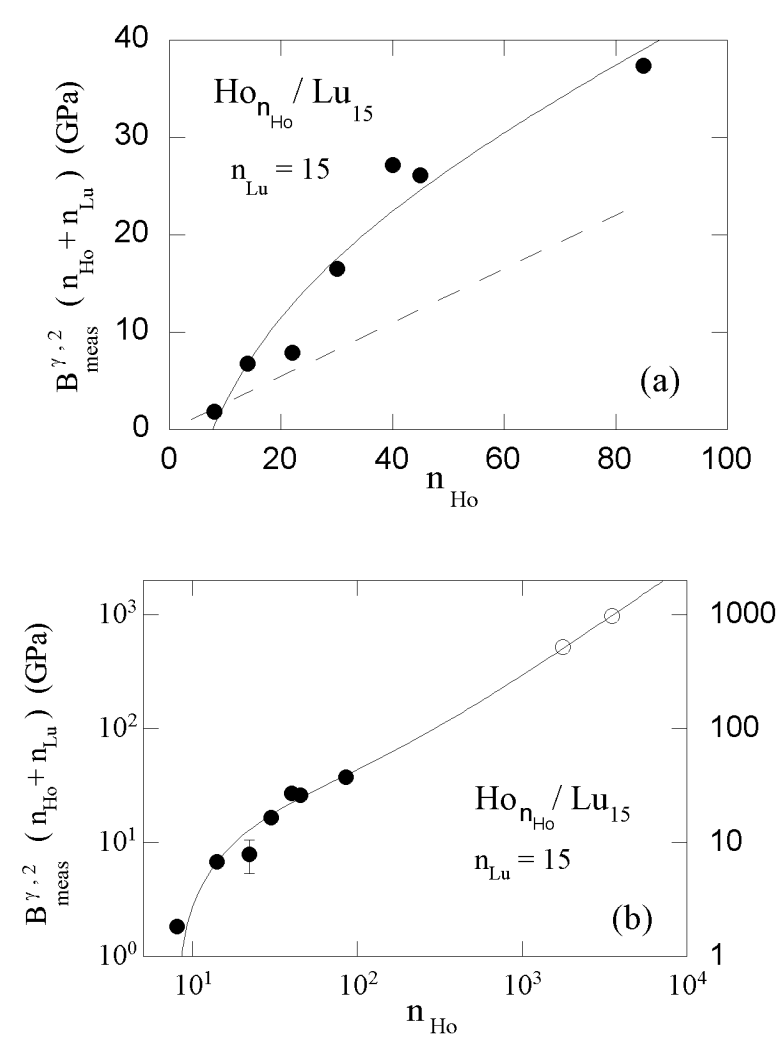

Fig. 7. $B_{\text {meas }}^{\gamma, 2}\left(n_{\mathrm{Ho}}+n_{\mathrm{Lu}}\right)$ for the series $\mathrm{Ho}_{n} / \mathrm{Lu}_{15}(\bullet)$, with $8<n<85$. The line is the function $B^{\gamma, 2} n_{\mathrm{Ho}}$, where $B^{\gamma, 2}$ is given by Eq. (34), with the fitting parameters $B_{v 0}^{\gamma, 2}=0.27 \mathrm{GPa}, \hat{B}_{\text {surf }}^{\gamma, 2}=$ - $7 \mathrm{GPa}$ and $D_{v}^{\gamma, 2}=-135 \mathrm{GPa}$, taking $e_{0}=\left(a_{\mathrm{Lu}}-a_{\mathrm{Ho}}\right) / a_{\mathrm{Lu}}=$ -0.017 . The dashed line is $B_{v 0}^{\gamma, 2} n_{\mathrm{Ho}}(a)$. Same as $(a)$ in a double-logarithmic plot, including the values for the two Ho films (O) $(b)$.

ence on the strain (this implies to have a non-linear dependence on the strain in the magnetoelastic energy). In this paragraph we will outline a model [36] which takes into account this fact, as well as the effect of the interfaces in the basal plane magnetoelastic stress in superlattices.

(a) Let us assume that the volume magnetoelastic parameter is, to first order in the strain,

$$
B_{v}^{\gamma, 2}=B_{v 0}^{\gamma, 2}+D_{v}^{\gamma} \varepsilon
$$

where $D_{v}^{\gamma}=\left(\partial B_{v}^{\gamma, 2} / \partial \varepsilon\right)_{\varepsilon=0}$ accounts for a possible modification of $B_{v}^{\gamma, 2}$ due to the epitaxial strain $\varepsilon$. This strain occurs when the two materials, A and B constituting the superlattice adjust their different bulk lattice parameters to a common one, having a intermediate value. If the lattice mismatch, defined in terms of the bulk's basal-plane $a$-lattice parameters of both materials as $e_{0}=\left(a^{\mathrm{B}}-a^{\mathrm{A}}\right) / a^{\mathrm{B}}$, is not too high (for $\mathrm{A}=\mathrm{Ho}$ and $B=\mathrm{Lu}$ we have $e_{0}=-0.017$, at $\left.10 \mathrm{~K}\right)$, it is reasonable to assume that $\mathrm{A}$ and $\mathrm{B}$ will accommodate their lattice parameters to a single value $a^{\mathrm{A}}=a^{\mathrm{B}}=a_{s l}$ (see end of
Sec. 3) and that no misfit dislocations will appear [37]. In Eq. (30) we have assumed isotropic basal plane strains, i.e., $\varepsilon_{x x}=\varepsilon_{y y}=\varepsilon$.

With the above hypotheses it is easy to relate the basal-plane strains of elements A and B through the mismatch $e_{0}$, obtaining

$$
\begin{aligned}
\varepsilon^{\mathrm{A}} & =\frac{a_{s l}^{\mathrm{A}}-a^{\mathrm{A}}}{a^{\mathrm{A}}}=\frac{a_{s l}^{\mathrm{B}}-a^{\mathrm{B}}+a^{\mathrm{B}}-a^{\mathrm{A}}}{a^{\mathrm{A}}} \simeq \\
& \simeq \frac{a_{s l}^{\mathrm{B}}-a^{\mathrm{B}}}{a^{\mathrm{B}}}+\frac{a^{\mathrm{B}}-a^{\mathrm{A}}}{a^{\mathrm{B}}}=\varepsilon^{\mathrm{B}}+e_{0} .
\end{aligned}
$$

The preceding relation allows to express the misfit elastic energy of a bilayer $\mathrm{A} / \mathrm{B}$ in terms of the epitaxial strain $\varepsilon^{\mathrm{A}}$. Minimization of that energy, considering free $x-y$ interfaces for the bilayer, with no stress component in the $z$ direction, gives us the equilibrium value of the basal plane epitaxial strain [33]:

$$
\varepsilon^{\mathrm{A}}=e_{0} \frac{t_{\mathrm{B}}}{k_{\mathrm{A}} k_{\mathrm{B}}^{-1} t_{\mathrm{B}}+t_{\mathrm{A}}},
$$

where $k_{\mathrm{A}, \mathrm{B}}$ are the following combinations of elastic constants for the A and B elements:

$$
k_{\mathrm{A}, \mathrm{B}}=c_{11}^{\alpha, \mathrm{A}, \mathrm{B}}\left(1-\frac{2 c_{13}^{\mathrm{A}, \mathrm{B}}}{c_{33}^{\mathrm{A}, \mathrm{B}}}\right)^{2}-
$$

$-\frac{1}{\sqrt{3}} c_{12}^{\alpha, \mathrm{A}, \mathrm{B}}\left[1-\left(\frac{2 c_{13}^{\mathrm{A}, \mathrm{B}}}{c_{33}^{\mathrm{A}, \mathrm{B}}}\right)^{2}\right]+\frac{1}{12} c_{12}^{\alpha, \mathrm{A}, \mathrm{B}}\left(1+\frac{2 c_{13}^{\mathrm{A}, \mathrm{B}}}{c_{33}^{\mathrm{A}, \mathrm{B}}}\right)^{2}$.

Now, Eq. (30) can be written in the form

$$
B_{v}^{\gamma, 2}=B_{v 0}^{\gamma, 2}+D_{v}^{\gamma, 2} e_{0} \frac{t_{\mathrm{B}}}{k_{\mathrm{A}} k_{\mathrm{B}}^{-1} t_{\mathrm{B}}+t_{\mathrm{A}}},
$$

where $k_{\mathrm{Ho}} / k_{\mathrm{Lu}}$ is close to 1 , for $\mathrm{A}$ being a rare-earth element and $\mathrm{B}$ being $\mathrm{Y}$ or $\mathrm{Lu}$.

(b) The interfacial contribution to the magnetoelastic stress is now treated similarly to the case of the interface anisotropy: the symmetry breaking at the surfaces or interfaces was related with the appearance of an additional term in the magnetocrystalline anisotropy, this term being proportional to the surface-to-volume ratio $1 / t$, where $t$ is the magnetic film (or block, in superlattices) thickness [38]. Then, considering the two surfaces or inter- 
faces per magnetic block, we add an interfacial term $2 B_{\text {surf }}^{\gamma, 2} / t_{\mathrm{A}}$ to the volume magnetoelastic stress of Eq. (33), obtaining

$$
B^{\gamma, 2}=B_{v 0}^{\gamma, 2}+\frac{2 \hat{B}_{\text {surf }}^{\gamma, 2}}{n_{\mathrm{A}}}+D_{v}^{\gamma, 2} e_{0} \frac{t_{\mathrm{B}}}{k_{\mathrm{A}} k_{\mathrm{B}}^{-1} t_{\mathrm{B}}+t_{\mathrm{A}}},
$$

where $\hat{B}_{\text {surf }}^{\gamma, 2}=B_{\text {surf }}^{\gamma, 2}\left(c_{\mathrm{A}} / 2\right)^{-1}$ is the value of the interfacial magnetoelastic stress expressed in units of energy per unit volume of the superlattice $\left(c_{\mathrm{A}} / 2\right.$ is the $(a, b)$-planes spacing for element A).

For the $\mathrm{Ho}_{n_{\mathrm{Ho}}} / \mathrm{Lu}_{n_{\mathrm{Lu}}}$ superlattices, substitution of Eq. (34) in Eq. (29) gives

$$
\begin{gathered}
B_{\text {meas }}^{\gamma, 2}= \\
=\frac{n_{\mathrm{Ho}}}{n_{\mathrm{Ho}}+n_{\mathrm{Lu}}}\left(B_{\mathrm{v} 0}^{\gamma, 2}+\frac{2 \hat{B}_{\mathrm{surf}}^{\gamma, 2}}{n_{\mathrm{Ho}}}+D_{v}^{\gamma, 2} e_{0} \frac{n_{\mathrm{Lu}}}{k_{\mathrm{Ho}} k_{\mathrm{Lu}}^{-1} n_{\mathrm{Ho}}+n_{\mathrm{Lu}}}\right)
\end{gathered}
$$

with $k_{\mathrm{Ho}} / k_{\mathrm{Lu}}=0.95$ and $e_{0}=-0.017$. Now, from Eq. (35), the advantage of plotting $B_{\text {meas }}^{\gamma, 2}\left(n_{\mathrm{Ho}}+n_{\mathrm{Lu}}\right)$ vs. $n_{\mathrm{Ho}}$ becomes clear. In Fig. 7 we show the fitting of the experimental values to the function $B^{\gamma, 2} n_{\mathrm{Ho}}$, where $B^{\gamma, 2}$ is given by Eq. (34). The parameters giving the best fit are $B_{v 0}^{\gamma, 2}=0.27 \mathrm{GPa}, \hat{B}_{\text {surf }}^{\gamma, 2}=$ $=-7 \mathrm{GPa}$ and $D_{v}^{\gamma, 2}=-135 \mathrm{GPa}$. In a $\log -\log$ plot (Fig. 7) we have included the values for two Ho films studied, also satisfying Eq. (35). Note that the obtained $B_{v 0}^{\gamma, 2}$ agrees with the value of $B^{\gamma, 2}$ for bulk holmium [34], which supports the proposed model. Interestingly, we have found a quite high value of the epitaxial stress increase of the unstrained volume stress parameter $\left(D_{\mathrm{v}}^{\gamma, 2} e_{0} n_{\mathrm{Lu}} /\left(n_{\mathrm{Lu}}+\right.\right.$ $\left.+n_{\mathrm{Ho}}\right) \approx 5.4 \cdot B_{v 0}^{\gamma, 2}$ for $n_{\mathrm{Ho}}=8$ ). Also, we have deduced a interfacial contribution to the magnetoelastic stress which is larger and of opposite sign to the volume stress $\left(2 \hat{B}_{\text {surf }}^{\gamma, 2} \approx-25 \cdot B_{\mathrm{v} 0}^{\gamma, 2} ;\right.$ e.g. $2 \hat{B}_{\text {surf }}^{\gamma, 2} / n_{\mathrm{Ho}} \approx$ $\approx-6.4 \cdot B_{\mathrm{v} 0}^{\gamma, 2}$ for $n_{\mathrm{Ho}}=8$ ). The interfacial stresses found in the present $\mathrm{Ho}_{n_{\mathrm{Ho}}} / \mathrm{Lu}_{15}$ superlattices are even higher than the highest values previously reported in $\mathrm{Cu} / \mathrm{Ni} / \mathrm{Cu}$ trilayers $\left(\hat{B}_{\text {surf }}^{\gamma, 2} \approx-6 \cdot B_{\text {v0 }}^{\gamma, 2}\right.$ ) [39].

\subsection{Temperature variation of the magnetoelastic stresses}

The study of the thermal dependence of the magnetoelastic stresses serves as a separate test of the previous analysis and the ensuing fitting parameters. For volume-originated distortions like $B_{v 0}^{\gamma, 2}$ and $D_{v}^{\gamma, 2}$, the standard Callen and Callen theory [27] predicts a temperature variation of the type $I_{5 / 2}\left[\mathcal{L}^{-1}(m(T))\right]$ (see Eq. (28)) . For the
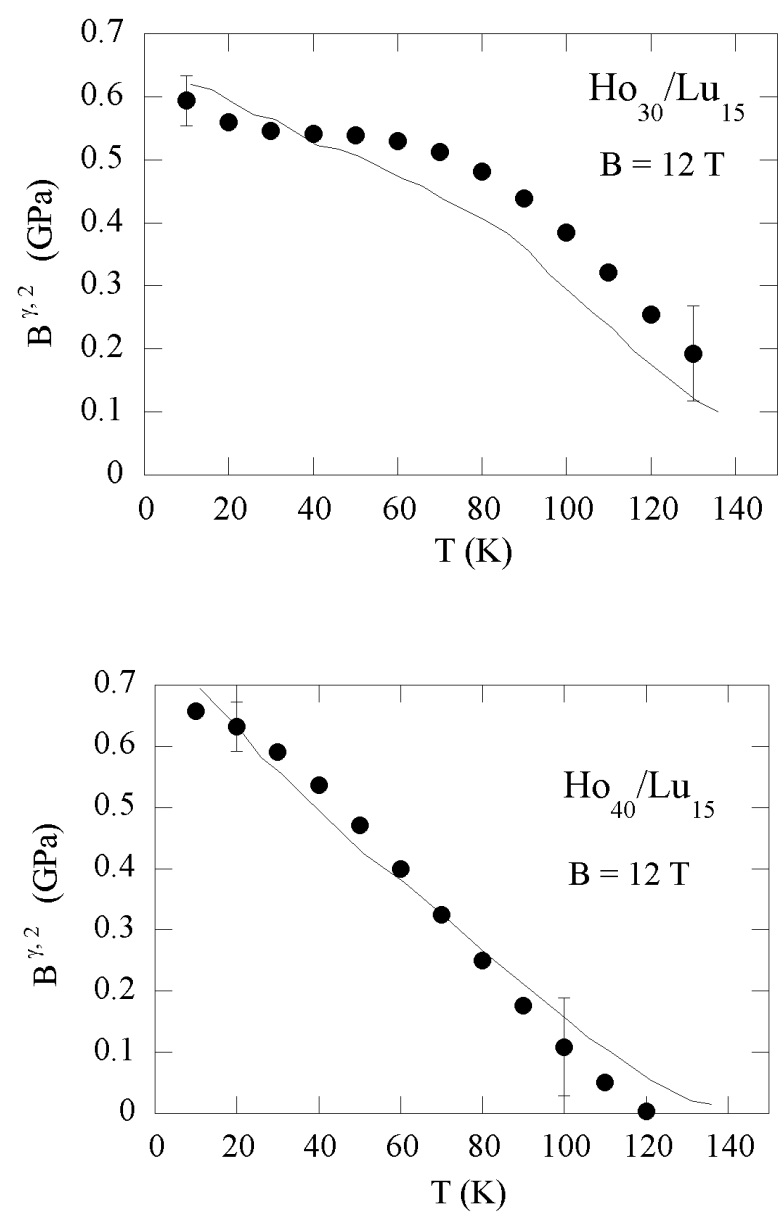

Fig. 8. Thermal dependence of $B^{\gamma, 2}$ for the $\mathrm{Ho}_{30} / \mathrm{Lu}_{15}$ and $\mathrm{Ho}_{40} / \mathrm{Lu}_{15}$ superlattices. The lines are theoretical fits using Eq. (36), which includes interface $B_{\text {surf }}^{\gamma, 2}$ and volume $D_{v}^{\gamma, 2}$ terms, the latter associated to the epitaxial strain.

interface magnetoelastic parameter $B_{\text {surf }}^{\gamma, 2}$, a dependence type $m^{4}(T)$ at low temperatures is predicted [40] if we assume that, at the interfaces, the spin dimensionality is $D=2$ (some interface anisotropy could account for this reduction in dimensionality). In the high temperature regime both volume and interface stress parameters should display the same thermal dependence, type $m^{2}(T)[27,40]$. Therefore, we have analyzed the temperature variation of $B^{\gamma, 2}$ by using the expression

$$
B^{\gamma, 2}=\left(B_{v 0}^{\gamma, 2}+D_{v}^{\gamma, 2} \varepsilon\right) \hat{I}_{5 / 2}\left[\mathcal{L}^{-1}(m)\right]+\frac{2 \hat{B}_{\text {surf }}^{\gamma, 2}}{n_{\text {Ho }}} m^{\alpha}
$$

where $\alpha=4$ at low temperatures and $\alpha=2$ at temperatures close to $T_{N}$.

In Fig. 8 we show the temperature variation of $B^{\gamma, 2}$ at $12 \mathrm{~T}$ for two of the studied samples, together with the theoretical fits obtained by using Eq. (36). 
Similar satisfactory results were obtained for the other samples, except for the superlattice with $n_{\mathrm{Ho}}=8$, were the fit was poorer. We employed the parameters previously deduced from the analysis of the Ho-thickness dependence of $B_{\text {meas }}^{\gamma, 2}\left(n_{\mathrm{Ho}}+n_{\mathrm{Lu}}\right)$ (see Fig. 7), allowing less than a $9 \%$ variation in their values. The interface contribution, varying as $m^{4}(T)$, was included below $50,80,55$, and $40 \mathrm{~K}$, for the superlattices with $n_{\mathrm{Ho}}=14,30,40$, and 45, respectively. These results support the validity of the magnetoelastic parameters obtained and confirm the single-ion crystal electric field origin for the magnetoelastic coupling in the $\mathrm{Ho} / \mathrm{Lu}$ superlattices.

\section{Magnetoelastic behavior of $\mathrm{Ho} / \mathrm{Y}$ superlattices}

We have carried out magnetoelastic stress measurements in the superlattices $\mathrm{Ho}_{10} / \mathrm{Y}_{n_{\mathrm{Y}}}$, where $n_{\mathrm{Y}}=10,30$, and 40 . In this case the interface contribution to $B^{\gamma, 2}$ remains unchanged, since the Ho layers thickness is kept constant. However, the influence of the epitaxial strain $\varepsilon$ on the volume magnetoelastic parameter is expected to be increased for increasing values of the $\mathrm{Y}$ layers thick-
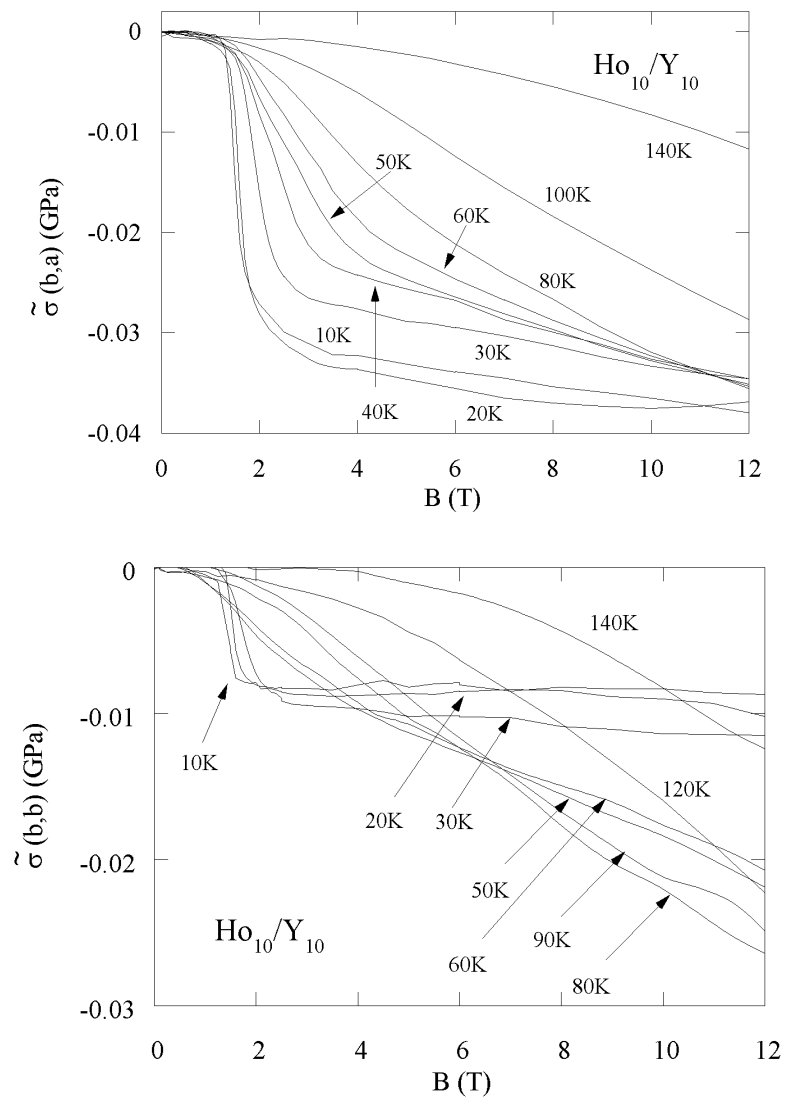

Fig. 9. Magnetoelastic stress isotherms $\tilde{\sigma}(b, a)$ and $\tilde{\sigma}(b, b)$ for the $\mathrm{Ho}_{10} / Y_{10}$ superlattice. ness (see Eq. (33). In Fig. 9 we show, as an example, the magnetoelastic stress isotherms for the $\mathrm{Ho}_{10} / \mathrm{Y}_{10}$ superlattice, where the transitions from helix to ferromagnetic phase at low temperatures are observed in the form of relatively abrupt changes in the stress; at higher temperatures, additional changes in the isotherm slope are associated with intermediary fan phases (the magnetization isotherms display such kind of changes at the same fields). Above $T_{N}$ the paramagnetic behavior is observed.

However, the most striking fact found in the present $\mathrm{Ho} / \mathrm{Y}$ series is the change of sign of the magnetoelastic stress parameter $B^{\gamma, 2}$. In Fig. 10 we show the $\tilde{\sigma}(b, a)$ and $\tilde{\sigma}(b, b)$ isotherms, at $10 \mathrm{~K}$ and $12 \mathrm{~T}$, for the $\mathrm{Ho}_{10} / \mathrm{Y}_{10}$ and $\mathrm{Ho}_{10} / \mathrm{Y}_{40}$ superlattices. For comparison, the values obtained for the $5000 \AA$ film are also shown. Note that, in order to compare the experimental values of $\tilde{\sigma}(i, j)$ corresponding to samples with different Ho volume fractions, we have plotted in Fig. 10 the measured stresses multiplied by $\left(t_{\mathrm{Y}}+t_{\mathrm{Ho}}\right) / t_{\mathrm{Ho}}$, according to Eq. (29) applied to the Ho $/ \mathrm{Y}$ case (we denote these normalized stresses as $\tilde{\sigma}_{\text {mag }}(i, j)$. The magnetoelastic stress $B^{\gamma, 2}=2\left[\tilde{\sigma}_{\text {mag }}(b, b)-\tilde{\sigma}_{\text {mag }}(b, a)\right]$ changes its sign along the series, as it is shown in Fig. 11 , where the $10 \mathrm{~K}$ and $12 \mathrm{~T}$ values of $B^{\gamma, 2}$ are plotted against the volume fraction of $\mathrm{Y}$. In this series, the interface contribution, inversely proportional to the Ho thickness, must be constant. Therefore, the plot in Fig. 11 reveals the dependence of the magnetoelastic stress with the epitaxial strain $\varepsilon$ on the Ho blocks, which is proportional to the $\mathrm{Y}$ volume fraction (see Eq.(32)). The line in Fig. 11 is a fit to the model Eq. (34), where $\mathrm{A}=\mathrm{Ho}$ and $\mathrm{B}=\mathrm{Y}, n_{\mathrm{Ho}}=10, k_{\mathrm{Ho}} / k_{\mathrm{Y}}=1.05$ and $e_{0}=0.023$. The parameters obtained from the fit are $B_{v 0}^{\gamma, 2}=0.27 \mathrm{GPa}$, $B_{\text {surf }}^{\gamma, 2}=2.16 \mathrm{GPa}$, and $D_{v}^{\gamma, 2}=-56 \mathrm{GPa}$ (the values

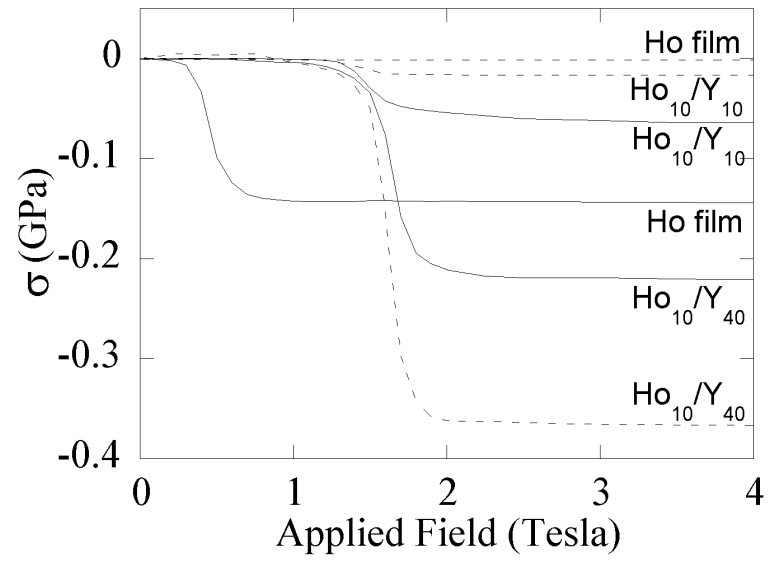

Fig. 10. Magnetoelastic stress isotherms $\tilde{\sigma}_{\text {mag }}(b, a)(-)$, and $\tilde{\sigma}_{\text {mag }}(b, b)(--)$ at 10 [4] $\mathrm{K}$ for the $\mathrm{Ho}_{10} / \mathrm{Y}_{10}$ and $\mathrm{Ho}_{10} / \mathrm{Y}_{10}$ superlattices and for the $5000 \AA$ Ho film. 


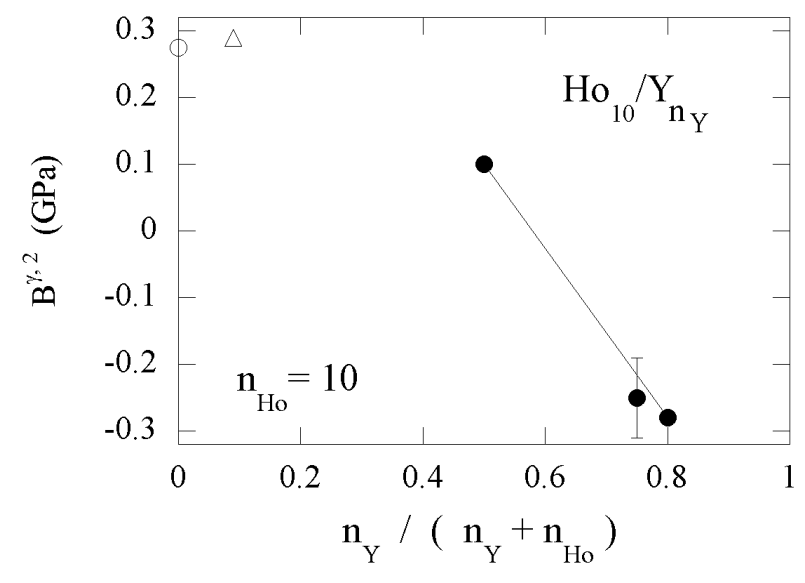

Fig. 11. Dependence of $B_{\text {mag }}^{\gamma, 2}$ with $n_{\mathrm{Y}} /\left(n_{\mathrm{Y}}+n_{\mathrm{Ho}}\right)$. The line is a fit to Eq. (34) (see text for details). The values for bulk Ho (○) and for the $5000 \AA$ A Ho film $(\Delta)$ are included.

for bulk Ho and for the $5000 \AA$ Ho film have not been included in the fit, although they are plotted in Fig. 11 to emphasize the change of the sign of $B^{\gamma, 2}$ in the $\mathrm{Ho}_{10} / \mathrm{Y}_{n_{\mathrm{Y}}}$ superlattices, for the film, $t_{\mathrm{Y}}=1000 \AA$, the $\mathrm{Y}$ seed thickness). For this series the constant interfacial contribution to the magnetoelastic stress is near two times the unstrained volume magnetoelastic stress $\left(2 \hat{B}_{\mathrm{surf}}^{\gamma, 2} / n_{\mathrm{Ho}} \approx 1.6 \cdot B_{\mathrm{V} 0}^{\gamma, 2}\right.$, with $n_{\mathrm{Ho}}=10$ ). The epitaxial stress contribution to the unstrained volume stress parameter depends on the $\mathrm{Y}$ thickness but it always has opposite sign to $B_{\mathrm{v} 0}^{\gamma, 2} \quad$ (e.g., $\quad D_{\mathrm{v}}^{\gamma, 2} e_{0} n_{\mathrm{Y}} /\left(n_{\mathrm{Y}}+n_{\mathrm{Ho}}\right) \approx-B_{\mathrm{v} 0}^{\gamma, 2}$, with $n_{\mathrm{Ho}}=10$ and for $\left.n_{\mathrm{Y}}=40\right)$.

The explanation of the change of sign of $B_{v}^{\gamma, 2}$ is another confirmation of the validity of the model assumptions, mostly the existence of a strain dependence of the volume magnetoelastic parameter, represented by the term $D_{v}^{\gamma, 2} \varepsilon$ in Eq. (34). The parameter $D_{v}^{\gamma, 2}$ is negative for both the $\mathrm{Ho} / \mathrm{Lu}$ and $\mathrm{Ho} / \mathrm{Y}$ studied series; however, the different sign of the lattice mismatch $e_{0}$ in both cases makes possible to observe the change of sign of $B^{\gamma, 2}$ only in the $\mathrm{Ho} / \mathrm{Y}$ series.

In order to understand the origin of the interface contribution to the magnetoelastic stress, a model of crystal electric field including screening due to the conduction band electrons has been proposed [41]. In this model, a Hartree-Fock-type dielectric constant and a Gaussian distribution, of half-width $b$, for the electronic charge density of the $\mathrm{Ho}^{3+}$ ions are assumed. The calculated values of $B_{\mathrm{v} 0}^{\gamma, 2}$ and $B_{\text {surf }}^{\gamma, 2}$, for the $\mathrm{Ho} / \mathrm{Lu}$ superlattices, agree in sign and magnitude with those determined in our experiments, indicating that both the volume and, more interestingly, the interface magnetoelastic stresses have their origin in the single-ion interaction with the distorted crystal electric field. The experimental values can be well reproduced if the Fermi energy of the interface, before epitaxy, is smaller than the volume one and if we take a density of states close to the obtained one from the bulk RAPW bandstructure calculations for Dy. The difference between the Fermi-energy values is simply, but quantitatively, explained assuming the formation of $\mathrm{Ho}^{3+}$ interface weakly localized electron states close to the Ho/Lu ideal contact plane [41]. However, two cautions are suggested: small systematic variations with $n_{\mathrm{Ho}}$ of the volume contribution $B_{\mathrm{v} 0}^{\gamma, 2}$ (and of $D_{v}^{\gamma} \varepsilon$ ) will result in variations of $B_{\text {surf }}^{\gamma, 2}$, although such an effect cannot be ascertained with our available experimental data; however, the sign and order of magnitude should probably be preserved, as both our volume and interface MEL parameters must be single-ion properties. Therefore, a more refined theory of the conduction electron band structure for the whole superlattice could predict changes in the Ho volume MEL stress extended to a certain number of layers within the Ho block.

\section{Competing anisotropies in the magnetoelastic behavior of Ho/Tm superlattices}

In this section we present a study of two Ho/Tm SL's: $\mathrm{Ho}_{8} / \mathrm{Tm}_{16}$ and $\mathrm{Ho}_{30} / \mathrm{Tm}_{16}$, nearly isomorphous to the corresponding $\mathrm{Ho}_{n} / \mathrm{Lu}_{15}$ samples analysed in Sec. 5. The differences when substituting Ho for $\mathrm{Lu}$ are, however, of fundamental importance. Now, the Tm «spacers» between Ho blocks are, not only magnetic, but also have the magnetization easy axis along c. In fact, bulk thulium has its magnetic structure longitudinally modulated along the $c$ axis below $T_{N}=58 \mathrm{~K}$ [42]. Its strong axial anisotropy hardly leaves that its magnetic moments been tilted out of the $c$ axis when a magnetic field is applied perpendicularly (within the basal-plane). This deviation is lesser than a $2 \%$ of the total moment at low temperatures and highfields, $\simeq 10 \mathrm{~T}$ [43]. However, a magnetic field applied parallel to the $c$ axis can break the modulated structure along this axis, and then gets a fully aligned magnetization state, $\simeq 2700 \mathrm{emu} / \mathrm{cm}^{3}$ at $0 \mathrm{~K}$, for magnetic field higher than $2.8 \mathrm{~T}$ [43].

Thus, having the $\mathrm{Ho} / \mathrm{Lu}$ system as a wellknown reference, we have chosen Ho/Tm SL's to study the presumable competition between the anisotropies of holmium and thulium layers in a SL system. By doing magnetization and MEL stress measurements we have analysed the differences which are observed when comparison with the hol- 
mium-lutecium results is made [44]. In this $\mathrm{Ho} / \mathrm{Tm}$ SL's, the analysis of the magnetization gives some insight about the kind of interaction existing between the Ho and Tm layers. However, the MEL stress measurements prove much better than magnetization whether the holmium layers or thulium ones dominate or not the magnetic behavior in Ho/Tm SL's. This is because, the MEL stress is a power of the magnetization $[27,40]$. In this way, a competition between the anisotropies of Ho and Tm ions can be clearly revealed by determining the MEL stresses in the Ho/Tm SL's. The different sign of the Ho and Tm anisotropies makes opposite the contribution to the magnetostriction from both ions, in particular, for the basal-plane cylindrical symmetry breaking MEL stress that we determine in these hexagonal-symmetry systems.

In Fig. 12 we show the $\tilde{\sigma}(b, b)$ and $\tilde{\sigma}(b, a)$ MEL stress isotherms, for the $\mathrm{Ho}_{8} / \mathrm{Tm}_{16}$ and $\mathrm{Ho}_{30} / \mathrm{Tm}_{16}$ superlattices. The MEL stress isotherms for the $\mathrm{Ho}_{8} / \mathrm{Lu}_{15}$ SL were shown in Fig. 6, and those for the $\mathrm{Ho}_{30} / \mathrm{Lu}_{15}$ are similar in their field dependence (saturation at relatively small magnetic fields, $H \geq 0.5 \mathrm{~T}$, and being more saturated when decreasing the temperature), although they have different saturation values.

As a first approach, and for comparison with the $\mathrm{Ho} / \mathrm{Lu}$ SL's, we can analyse the MEL stress data obtained in the Ho/Tm samples assuming that the $\mathrm{Tm}$ moments do not leave their magnetization easy axis, even under the action of the maximum applied field of $12 \mathrm{~T}$. In this way we obtain the $B^{\gamma, 2}$ MEL parameter, which is shown as a function of the temperature in Fig. 13, for the two Ho/Tm SL's studied, together with the $B^{\gamma, 2}$ values for the isomorphous $\mathrm{Ho} / \mathrm{Lu}$ samples.

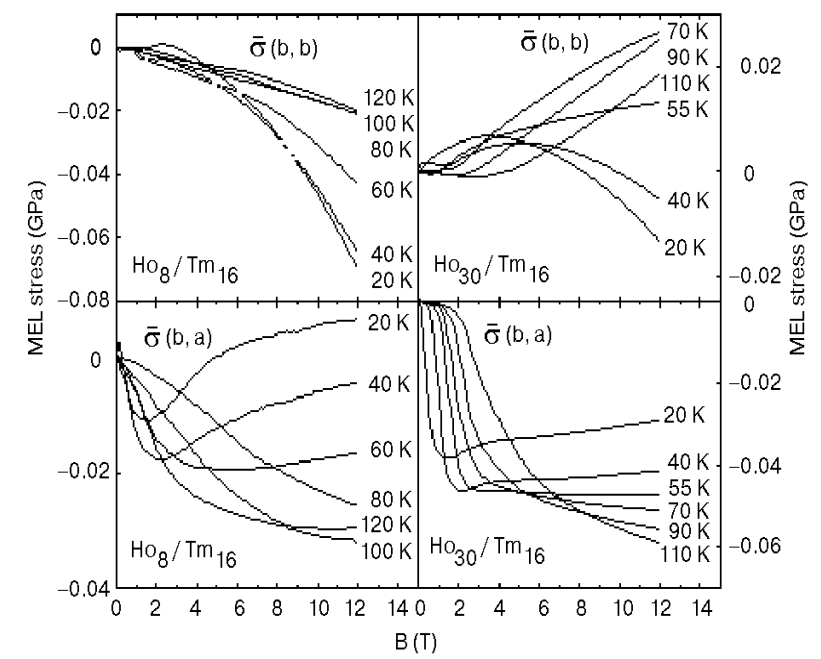

Fig. 12. Magnetoelastic stress isotherms for the $\mathrm{Ho}_{8} / \mathrm{Tm}_{16}$ and $\mathrm{Ho}_{30} / \mathrm{Tm}_{16}$ superlattices.
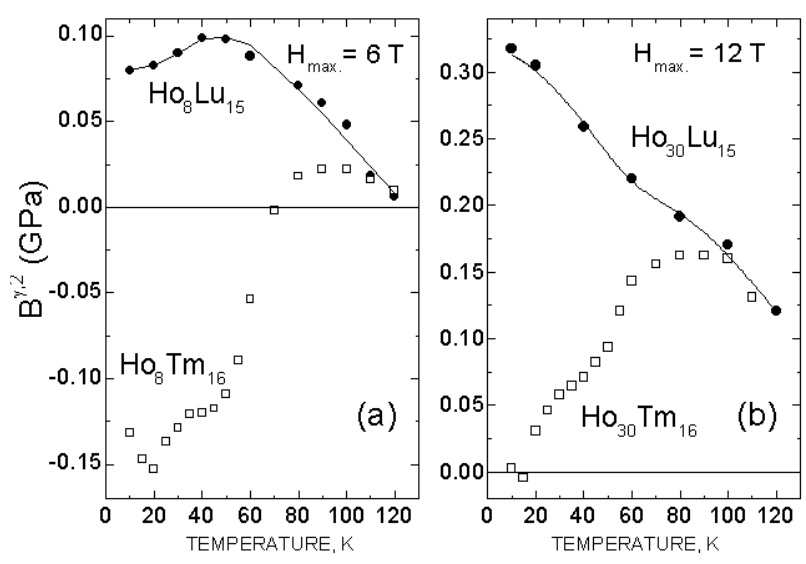

Fig. 13. Thermal dependence of $B^{\gamma, 2}$ for the $\mathrm{Ho}_{8} / \mathrm{Tm}_{16}$ and $\mathrm{Ho}_{8} / \mathrm{Lu}_{15}$ SL's $(a)$ and the $\mathrm{Ho}_{30} / \mathrm{Tm}_{16}$ and $\mathrm{Ho}_{30} / \mathrm{Lu}_{15}$ SL's $(b)$.

The values of $B^{\gamma, 2}$ for the Ho/Tm SL's are positive at high temperatures and very close to the $\mathrm{Ho} / \mathrm{Lu}$ ones, showing a broad peak at around $80-90 \mathrm{~K}$. Below $80 \mathrm{~K}, B^{\gamma, 2}$ start to decrease, reaching a maximum slope on decreasing the temperature at $\simeq 60 \mathrm{~K}$, and undergoing a change of sign at a certain temperature, which is $60 \mathrm{~K}$ for $\mathrm{Ho}_{8} / \mathrm{Tm}_{16}$ and $20 \mathrm{~K}$ for $\mathrm{Ho}_{30} / \mathrm{Tm}_{16}$. Considering the singleion CEF origin of the $B^{\gamma, 2}$ MEL stress, this strong deviation towards negative values is interpreted as a result of the competition of the $\mathrm{Ho}$ and $\mathrm{Tm}$ anisotropies. Moreover, the effect of the Tm layers is much more intense in $\mathrm{Ho}_{8} / \mathrm{Tm}_{16} \mathrm{SL}$ than in $\mathrm{Ho}_{30} / \mathrm{Tm}_{16}$, where the basal-plane anisotropy of holmium blocks dominates the $B^{\gamma, 2}$ MEL stress, making the values more positive for this SL, where the thickness of holmium layers is high enough with respect to the thulium ones. This is in good agreement with the magnetization results too [44]. So, our MEL stress experiments clearly indicate that the Tm moments are tilted out from the $c$ axis when a magnetic field is applied within the basal plane of the hcp structure (otherwise the values of $B^{\gamma, 2}$ would be nearly the same as for the Ho/Lu SL's), this effect being larger in the case of the $\mathrm{Ho}_{30} / \mathrm{Tm}_{16}$ sample than for the $\mathrm{Ho}_{8} / \mathrm{Tm}_{16} \mathrm{SL}$. Nevertheless, experiments in higher magnetic fields are needed to attempt the full saturation of these competing anisotropy SL's, and, in this way, to be able to obtain separately the Tm and Ho MEL stress parameters [44].

\section{Magnetoelastic behavior of Dy / Y and $\mathrm{Er} /$ Lu superlattices}

In dysprosium based SL's the determination of the easy basal plane cylindrical symmetry breaking 
magnetoelastic stress, $B^{\gamma, 2}$, is of paramount interest, inasmuch as it decisively helps to drive the spontaneous helical $(\mathrm{H})$ ferromagnetic $(\mathrm{F})$ transition in bulk Dy, at $T_{c}>85 \mathrm{~K}$ [7]. However, it was observed than in $\mathrm{Dy} / \mathrm{Y}$ superlattices, grown along the $\mathrm{c}$ axis, the $\mathrm{Y}$ block tensile stress suppresses the $\mathrm{H}-\mathrm{F}$ transition [1].

In bulk Er the c axis is easy, and the magnetic structure changes from paramagnetic to sinusoidal at $T_{N 1}>85 \mathrm{~K}$, to the (a, c) plane elliptically cycloidal structures at $T_{N 2}>52 \mathrm{~K}$ and to ferroconical phase of wave vector $(5 / 21) \mathbf{c}^{*}$ at $T_{c}>20 \mathrm{~K}$ [45,46]. To our knowledge no magnetic phase diagrams have been traced for $\mathrm{Er} / \mathrm{Lu}$ superlattices.

We have carried out magnetoelastic stress measurements in the superlattices $\left(\mathrm{Dy}_{n} / \mathrm{Y}_{15}\right)$ and $\left(\mathrm{Er}_{m} / \mathrm{Lu}_{10}\right)$, with $n=5,15,25$, and $m=10,20,30$ atomic planes, from $10 \mathrm{~K}$ and in applied magnetic fields up to $B=12 \mathrm{~T}$; the magnetic field was applied within the basal plane, along the a easy axis, for both series.

In Fig. 14 we present, as an example, the $\tilde{\sigma}(a, b)$ and $\tilde{\sigma}(a, a)$ isotherms for $\mathbf{B} \| \mathbf{a}$, for the $\left[\mathrm{Dy}_{25} / \mathrm{Y}_{15}\right]_{50}$ SL. We notice changes in slope for certain fields that we tentatively ascribe to the $\mathrm{H}$-fan (FN) and to the FN-F transitions. These fields correspond well with the observed ones on the magnetization isotherms. As we may observe, saturation is practically accomplished at $12 \mathrm{~T}$ for $T<T_{N}$. This indicates that we are measuring a MS of crystal electric field origin, once the sample is $\mathrm{F}$. For the Er SL's in Fig. 14, $b$ we present, as an example, the same kinds of isotherms for the $\mathrm{Er}_{30} / \mathrm{Lu}_{10} \mathrm{l}_{40} \mathrm{SL}$, where we observe: three changes in slope for $T<T_{c}$, two for $T_{c}<T<40 \mathrm{~K}$, and no features for $40 \mathrm{~K}<T<T_{N 1}$, if we tentatively ascribe the same phases of the bulk to the SL $[45,46]$. Similar changes in slope were observed in the magnetization isotherms at about the same fields for $T<T_{c}$, although for the range $40 \mathrm{~K}<T<T_{N 1}$, a slope change is observed at about $2 \mathrm{~T}$.

According to bulk neutron diffraction measurements and mean field calculations $[45,46]$ we tentatively ascribe the transitions for $T<T_{c}$ as follows: The transition at $H_{c 1} \cong 3 \mathrm{~T}$ could be to a conical-fan structure of wave vector $(1 / 4) c^{*}$, which, at $H_{c 2} \cong$ $\cong 8 \mathrm{~T}$, transforms to an FN structure around the $a$ axis. Finally, the transition at $H_{c 3} \cong 12 \mathrm{~T}$ could be toward an $\mathrm{F}$ one along the $a$ axis. Notice that only the transition at $H_{c 1}$ is abrupt. For the range $T_{c}<T<40 \mathrm{~K}$, we observe changes of slope at about $1,2,5$, and $12 \mathrm{~T}$. If we tentatively translate from the bulk [45] these fields would respectively correspond to transitions to commensurable cycloi- dal structures of wave vectors (in $c^{*}$ units) $6 / 23$ and $4 / 15$, a fan $2 / 7$ about the basal plane, and toward an $\mathrm{F}$ phase along $\mathbf{B}$.

If we analyse the thickness dependence of the magnetoelastic stress of the Dy/Y SL's as we did for the $\mathrm{Ho} / \mathrm{Lu}$ SL's, we obtain the values: $B_{y 0}^{\gamma, 2}=$ $=0.85 \mathrm{GPa}, \quad D_{y}^{\gamma}=5.2 \cdot 10^{2}$ and $B_{\text {surf }}^{\gamma, 2} /(c / 2)=$ $=-22.8 \mathrm{GPa}$ [5] (note that the SL with $n=5$ can only be included in the analysis duplicating the value of $D_{v}^{\gamma}$, which indicates that the SL's with very thin Dy blocks are more sensitive to epitaxial strain variations). The effective interface magnetoelastic stress $2 B_{\text {surf }}^{\gamma, 2} t_{\mathrm{Dy}}$ is strong compared to the volume term, up to about one order of magnitude larger and of the opposite sign.
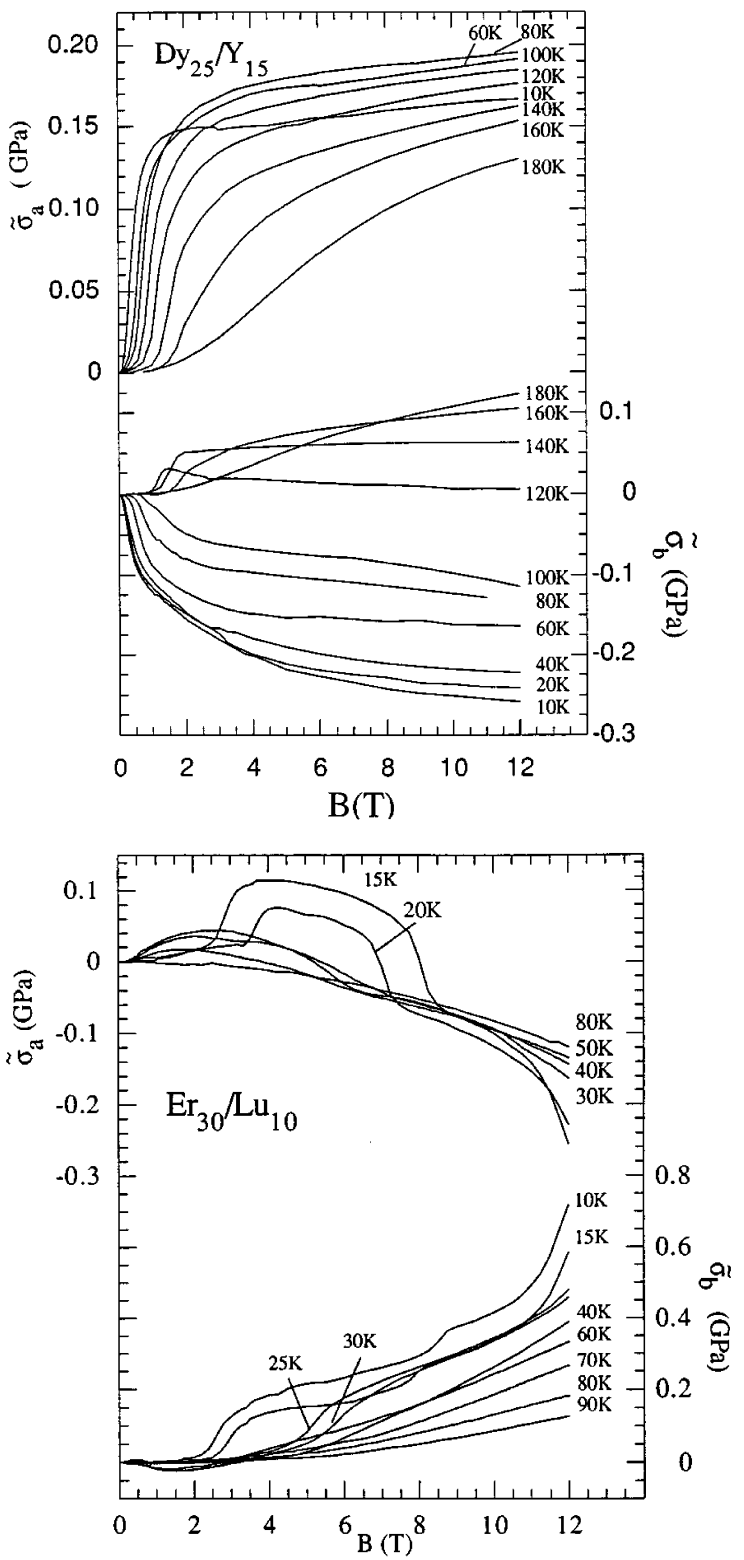

Fig. 14. Magnetoelastic stress isotherms for the SL's $\left[\mathrm{Dy}_{25} / \mathrm{Y}_{15}\right]_{50} \quad(a)$ and $\left[\mathrm{Er}_{30} / \mathrm{Y}_{10}\right]_{40} \quad(b), \quad \tilde{\sigma}_{a} \equiv \tilde{\sigma}(a, b)$ and $\tilde{\sigma}_{b} \equiv \tilde{\sigma}(a, a)$ correspond to SL clamping along the $a$ and $b$ axes. 


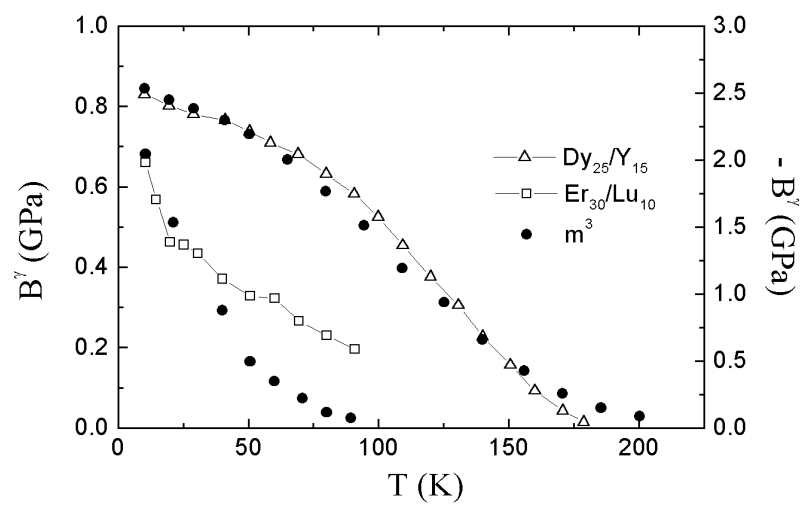

Fig. 15. Comparison of $B^{\gamma, 2}(T)$ with $m^{3}(T)$ at $12 \mathrm{~T}$, where $m$ is the reduced magnetization, for $\mathrm{Dy}_{25} / \mathrm{Y}_{15}$ and $\mathrm{Er}_{30} / \mathrm{Lu}_{10}$ SL's.

Another test of the CEF origin for the $B^{\gamma, 2}$ is to check if it scales with the reduced saturation (at $B=12 \mathrm{~T})$ magnetization $m=M(T) / M(0)$ as $m^{3}$, according to the standard theory of magnetostriction [27]. In Fig. 15 we compare for the $\mathrm{Dy}_{25} / \mathrm{Y}_{15}$ SL the thermal variation of $B^{\gamma, 2}(12 \mathrm{~T})$ with the one for $m^{3}(12 \mathrm{~T})$; the agreement is satisfactory. For the $\mathrm{Er}_{n} / \mathrm{Lu}_{10}$ SL's we have done a study fully similar to the one above for the $\mathrm{Dy} / \mathrm{Y}$ SL's. However, as it can be seen in the isotherms plotted in Fig. 14, $b$ the magnetoelastic stresses are far away from saturation, as it happens with the magnetization [33]. Thus, the analysis of the experimental results in this case is inconclusive (see Fig. 15 and Ref. 5). Magnetoelastic stress experiments in larger fields, up to $30 \mathrm{~T}$, which are needed to saturate this SL's within the growing plane, are in progress.

\section{Magnetoelastic behavior of (110) $\mathrm{TbFe}_{2} / \mathrm{YFe}_{2}$ bilayers}

For the $\mathrm{TbFe}_{2} / \mathrm{YFe}_{2}$ bilayers, the MEL stress measurements were performed between $10 \mathrm{~K}$ and $\mathrm{RT}$ and the magnetic field, up to $12 \mathrm{~T}$, was applied within the growing plane (temperatures below $10 \mathrm{~K}$ were avoided because of the diamagnetic behavior of the $\mathrm{Nb}$ buffer). In Fig. 16 we show, as an example, isotherms corresponding to the $\mathrm{TbFe}_{2}$ $(600 \AA) / \mathrm{YFe}_{2}(1000 \AA)$ bilayer, for the stresses: $\tilde{\sigma}([\overline{1} 10],[001]), \tilde{\sigma}([\overline{1} 10],[\overline{1} 10])$ and $\tilde{\sigma}([\overline{1} 11],[1 \overline{1} 2])$ (see Eqs. (16), (17), and in Sec. 2.2.2). For the sake of clarity, and because of the similarity between the different isotherms for a given sample, we have only plotted the isotherms obtained at the lowest and the highest temperatures of the measuring range. At the beginning, and before starting each measurement, we have demagnetized the sample. Due to the high Curie temperature of

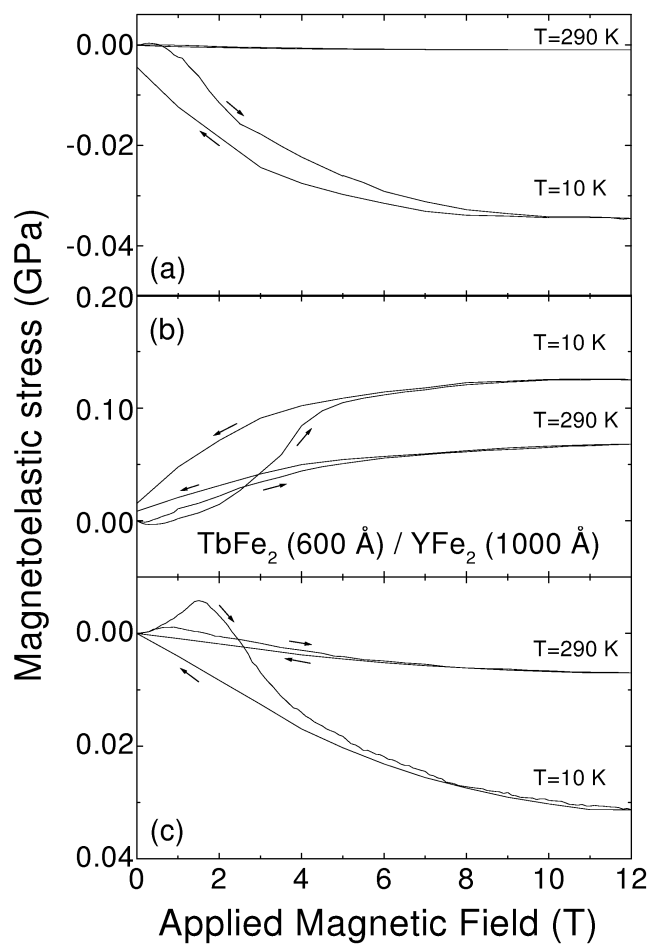

Fig. 16. The magnetoelastic stress isotherms for $\mathrm{TbFe}_{2}(600 \AA) / \mathrm{YFe}_{2}(1000 \AA)$ bilayer: $\sigma([\overline{1} 10],[001]) \quad(a)$; $\sigma([\overline{1} 10],[\overline{1} 10])(b)$, and $\sigma([\overline{1} 11],[1 \overline{1} 2])(c)$. For the sake of simplicity we only represent the RT and $10 \mathrm{~K}$ isotherms.

$\mathrm{TbFe}_{2}$, of about $696 \mathrm{~K}$, it was unfeasible with our experimental set-up to get any virgin state by heating up first the sample above $700 \mathrm{~K}$ and, then, cooling it down to get the initial demagnetized state. Moreover, at these high temperatures the bilayer structure would be destroyed due to the diffusion between the layers. Instead of this, we have performed, at the measuring temperature, hysteresis cycles with decreasing amplitude of the maximum applied magnetic field in each cycle, to get a macroscopically demagnetized sample. From the MEL stress isotherms, we can distinguish two kinds of magnetoelastic behavior, depending on the applied magnetic field direction. For those measurements where the magnetic field is parallel to [1111] direction (see Fig. 16,c), the MEL isotherms show a large hysteresis with coercive field values very close to those observed in the magnetisation measurements [18]. On the contrary, when the field is applied along [1110] direction, the MEL curves hardly show hysteresis, the fields for zero MEL stress crossing being even smaller than the corresponding ones obtained from magnetisation hysteresis measurements. In all the cases the coercive fields increase on reducing the thickness of $\mathrm{TbFe}_{2}$ layer. In spite of these differences, it is important to note that all the loops have approximately the same 
«closing field», which has very large values $(\approx 6 \mathrm{~T}$ at RT). Moreover, at the maximum applied field of $12 \mathrm{~T}$, the $\tilde{\sigma}(\alpha, \beta)$ MEL stresses do not show saturation at all. This lack of saturation even occurs when the magnetic field is applied along the easy [1111] direction, which, however, seems to show the best approach to the saturation. To some extent, magnetostriction is more sensible to show the lack of the full saturation than magnetization, a usual fact. Therefore, since the magnetization is indeed saturated at $12 \mathrm{~T}$ [18], we will consider the MEL stress values at $12 \mathrm{~T}$ as corresponding to a saturated regime.

\subsection{Magnetoelastic stress parameters}

We have obtained the $b_{0}, b_{1}$, and $b_{2}$ MEL stress parameters as explained in Sec. 2.2.2. Their thermal dependences for the different $\mathrm{TbFe}_{2}(t) / \mathrm{YFe}_{2}$ bilayers are shown in Fig. 17 (the $t=300 \mathrm{~A}$ sample is the only one of the series which does not show a measurable MEL stress). We can see a smooth but monotonous decrease of all the parameters on rising up the temperature, and also that the values of $b_{0}$ are about one order of magnitude smaller than those of $b_{1}$ and $b_{2}$. Notice that, because of the selected applied field and MEL stress directions, it has been possible to determine directly all the second order MEL stress parameters in the present bilayers.

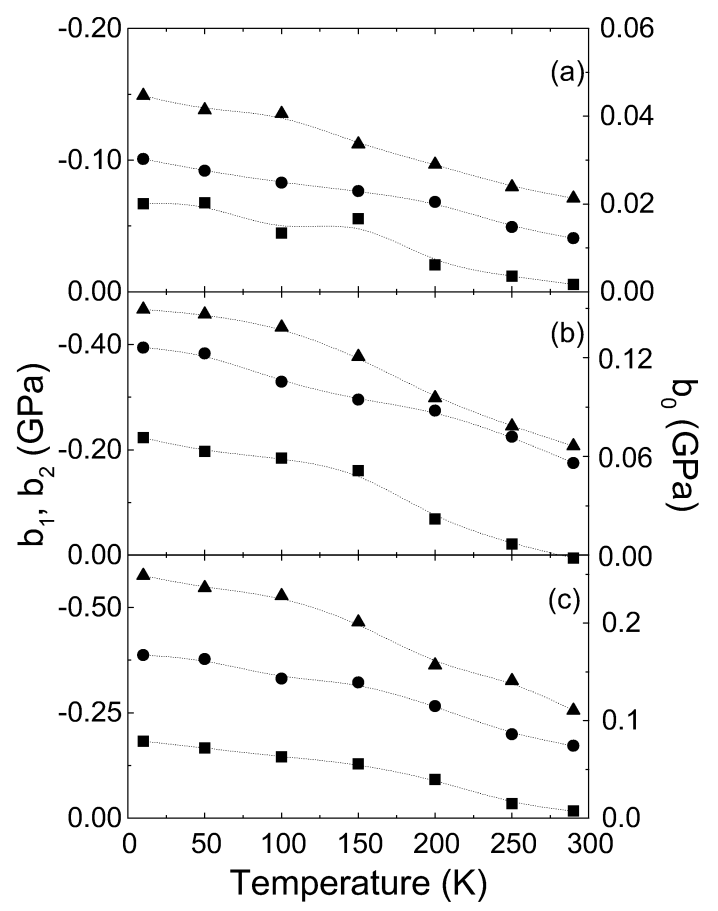

Fig. 17. The temperature dependence of the MEL stress parameters: $b_{0}$ (isotropic MEL stress) (ם); $b_{1}$ (tetragonal MEL

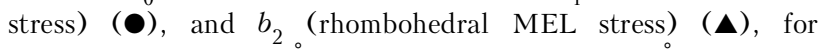
$\mathrm{TbFe}_{2}(t) / \mathrm{YFe}_{2}(1000 \AA)$ bilayers at different $t$, ̊: $600(a)$; $1000(b) ; 1300(c)$.
However, $b_{0}$ was never determined before in bulk $\mathrm{TbFe}_{2}$, and therefore, we cannot compare its value with those ones for the $\mathrm{TbFe}_{2} / \mathrm{YFe}_{2}$ bilayers.

We also can observe that $b_{1}$, for $\mathrm{TbFe}_{2}(1300 \AA) / \mathrm{YFe}_{2}(1000 \AA)$ sample and $\mathrm{TbFe}_{2}(1000 \AA) / \mathrm{YFe}_{2}(1000 \AA)$ sample are very close in value, $\simeq-0.4 \mathrm{GPa}$ at $10 \mathrm{~K}$ and $12 \mathrm{~T}$, but for $\mathrm{TbFe}_{2}(600 \AA) / \mathrm{YFe}_{2}(1000 \AA)$ it is about $25 \%$ smaller than in the others. This could indicate that $b_{1}$ tends to saturate for increasing $\mathrm{TbFe}_{2}$ block thickness in the bilayer. However, from anisotropic magnetostriction, $\lambda_{t}^{s}$, measurements in polycrystalline $\mathrm{TbFe}_{2}$ and from measurements in crystalline $\mathrm{TbFe}_{2}$, and using the relationship $\lambda_{t}^{s}=(3 / 5) \lambda_{[100]}+$ $+(9 / 10) \lambda_{[1 \overline{1} 1]}[47-51]$, we have estimated a $0 \mathrm{~K}$ value of $b_{1} \simeq-0.11 \mathrm{GPa}$ for bulk $\mathrm{TbFe}_{2}$, which is less than half of those obtained for the $t=1000 \AA$ and $1300 \AA$ studied bilayers. The origin of this enhancement of MEL stress in the bilayers will be analysed in more detail later.

It is interesting to note that $b_{2}$ is $74 \%$ of the bulk's value for $\operatorname{TbFe}_{2}(1300 \AA) / \mathrm{YFe}_{2}(1000 \AA)$, at RT and $12 \mathrm{~T}$, whereas it is only a $55 \%$ of it in the case of $\mathrm{TbFe}_{2}(1300 \AA)$ on $\mathrm{NbFe}^{-} \varphi[4]$. This would indicate that the use of $\mathrm{NbFe}^{-} \varphi(15 \AA)$ as buffer instead of $\mathrm{YFe}_{2}(1000 \AA)$ decreases the value of the MEL stress, $b_{2}$, in the $(110) \mathrm{TbFe}_{2}(1300 \AA)$ thin films. The reason why this happens is not very well understood at the present, although the epitaxial strain should be playing an important role. The strain originated by the epitaxial growth of the $1300 \AA$ thick $\mathrm{TbFe}_{2}$ layer onto the $1000 \AA$ thick $\mathrm{YFe}_{2}$ buffer is $\simeq-0.54 \%$, at RT [31] and, when the buffer is a $15 \AA$ thick $\mathrm{NbFe}^{-} \varphi$ layer, the epitaxial strain increases up to $\simeq-0.64 \%[30,52]$. Therefore such a small misfit strain reduction changes substantially the rhombohedral, $b_{2}$, MEL parameter. This could also happen with the observed increase of $\left|b_{2}\right|$ (see Fig. 16) when $t$ increases because, as the $\mathrm{TbFe}_{2}$ block thickness increases, the shear strain, $\varepsilon_{x y}$, decreases [31]. Now, and since we have information about the in-plane strains in the bilayers, we can perform the same kind of analysis as we did for the RE SL's, although modified for the current cubic symmetry, to obtain information about the origin of the MEL coupling in the present systems. So, for $b_{2}$ we can write

$$
b_{2}=b_{2}^{v}+\frac{b_{2}^{s}}{t}=\left(b_{2}^{v 0}+d_{2}^{v} \varepsilon_{x y}^{0}\right)+\frac{b_{2}^{s}}{t},
$$

where $b_{2}^{v 0}$ is the main volume contribution, $d_{2}^{v}=$ $=\left(\partial b_{2} / \partial \varepsilon_{x y}^{0}\right)$ taking into account the non-linear effects on the MEL energy of Eq. (8). This strain, 
within the elastic regime (see Sec. 5.1), for the cubic symmetry reads

$$
\varepsilon_{x y}^{0}=\varepsilon_{0} \frac{1000 \AA}{\beta_{2} t+1000 \AA},
$$

where $\varepsilon_{0}(\simeq-0.7 \%)^{31}$, is $\left(a_{\perp}^{\mathrm{TbFe}_{2}}-a_{\perp}^{\mathrm{YFe}}{ }_{2}\right) /\left\langle a_{\perp}\right\rangle$, being $a_{\perp}^{\mathrm{REFe}_{2}}$ the lattice parameters along [220] and $\beta_{2} \equiv$ $\equiv c_{44}^{\mathrm{A}} / c_{44}^{\mathrm{B}}$, where $c_{44}^{\mathrm{A}, \mathrm{B}}$ are the shear elastic constants for $\mathrm{A} \equiv \mathrm{TbFe}_{2}$ and $\mathrm{B} \equiv \mathrm{YFe}_{2}$. Notice that the value of $\beta_{2} \simeq 0.2$ has been deduced from the $\varepsilon_{x y}^{0}$ experimental results (only at RT [30,31] by using Eq. (38) (remember that $\varepsilon_{x y}^{0}$ depend on $t$ and their values have been given in Sec. 3.3). The small value obtained for $\beta_{2}(<<1)$ could indicate that the elastic regime assumption that we are taking for granted would not be valid for large t values (we should note that, if we consider a possible dislocation energy, we would arrive to a dependence with the thickness similar to that appearing in Eq. (38) [53,54]; so, both contributions, elastic and dislocation energy, should be taken into account and this could change the value obtained for $\beta_{2}$ ). The last term in Eq. (37) is the Neel interface contribution to the stress. In Fig. 18, $b$ we plot the RT values of $b_{2}$ versus $\mathrm{TbFe}_{2}$ thickness, as obtained from Fig. 17 . The small value of $b_{2}$ for the $\mathrm{TbFe}_{2}(300 \AA) / \mathrm{YFe}_{2}$

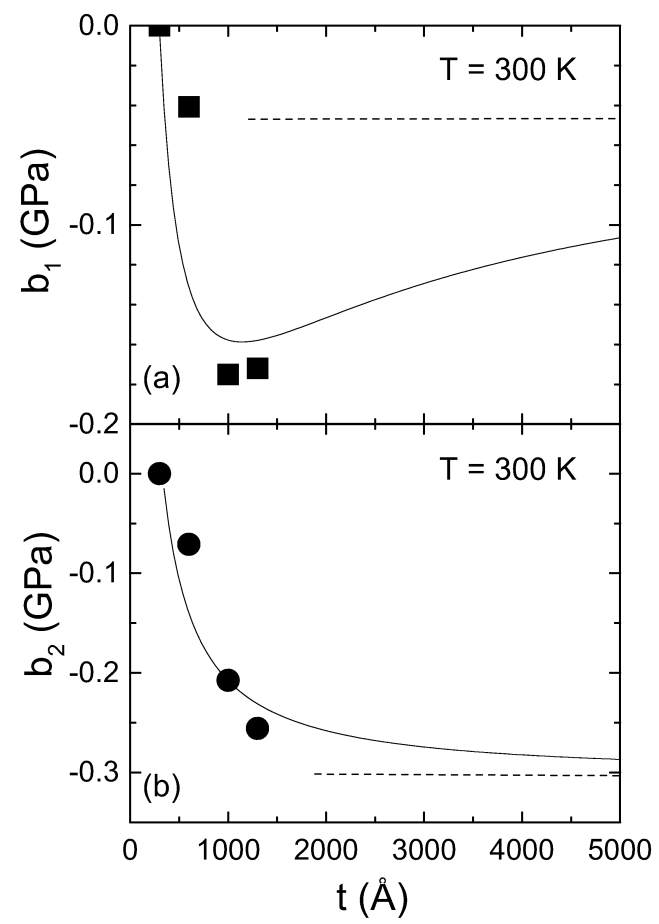

Fig. 18. $\mathrm{TbFe}_{2}$ block thickness dependence of the tetragonal MEL stress parameter, $b_{1}(a)$, and the rhombohedral MEL stress, $b_{2}(b)$, at RT. The continuous lines represent the best fits using the Eqs. (39) and (37). The horizontal dotted lines are the values of $b_{1}$ and $b_{2}$ for bulk $\mathrm{TbFe}_{2}$. Notice the asymptotic approximation to these values of $b_{1}$ and $b_{2}$ for the bilayers.
(1000 A) bilayer [17], and the bulk $\mathrm{TbFe}_{2}$ value, $b_{2}^{v 0}=-0.307 \mathrm{GPa}[47,48]$ are also shown. We represent in the same Figure the fit of these values by the model expression given in Eq. (37). The fit is good regardless the reduced number of experimental points available. The parameters giving the best fit are: $d_{2}^{v} \varepsilon_{0}=0.0158 \mathrm{GPa}$ and $b_{2}^{s}=98 \mathrm{GPa} \cdot \stackrel{\circ}{\mathrm{A}}$, and so $d_{2}^{v}=-2.3 \mathrm{GPa}$. For instance, for $t=1000 \AA$, the contributions to $b_{2}$ amount: $b_{2}^{v}=-0.313 \mathrm{GPa}$ and $b_{2}^{s} / t=-0.1 \mathrm{GPa}$. We see that the overall volume MEL stress is practically the same as the bulk one and three times the interfacial contribution. Clearly non-linear MEL effects on $b_{2}$ (rhombohedral striction) are small for our bilayers.

The same kind of analysis can be applied to $b_{1}$, i.e.,

$$
b_{1}=b_{1}^{v}+\frac{b_{1}^{s}}{t}=\left(b_{1}^{v 0}+d_{1}^{v} \varepsilon_{x x}^{0}\right)+\frac{b_{1}^{s}}{t}
$$

where $d_{1}^{v}=\left(\partial b_{1} / \partial \varepsilon_{x x}^{0}\right)_{\mathrm{eq}}$ with $\varepsilon_{x x}^{0}$ the misfit strain. This strain is

$$
\varepsilon_{x x}^{0}=\left(+\frac{\varepsilon_{0}}{2}-e_{0}\right) \frac{1000 \AA}{\beta_{1} t+1000 \AA}-\frac{\varepsilon_{0}}{2} \mathcal{K},
$$

being $e_{0}$ the lattice mismatch for the bulk $\mathrm{TbFe}_{2}$ and $\mathrm{YFe}_{2}\left(e_{0} \simeq-0.19 \%\right)$ and $\mathcal{K}_{0} \equiv 1000 \AA t\left[c_{11}^{\mathrm{A}} c_{12}^{\mathrm{B}}-\right.$ $\left.-c_{11}^{\mathrm{B}} c_{12}^{\mathrm{A}}\right] /\left\{\left[t\left(c_{011}^{\mathrm{A}}+2 c_{12}^{\mathrm{A}}\right)+1000 \AA\right.\right.$ $\left.\left.\left.-c_{12}^{\mathrm{A}}\right)+1000 \AA\left(c_{11}^{\mathrm{B}}-c_{12}^{\mathrm{B}}\right)\right]\right\} \quad$ and $\beta_{1} \equiv\left(c_{11}^{\mathrm{A}}+\right.$ $\left.+2 c_{12}^{\mathrm{A}}\right) /\left(c_{11}^{\mathrm{B}}+2 c_{12}^{\mathrm{B}}\right)$, where $c_{1, j}^{\mathrm{A}, \mathrm{B}}(j=1,2)$ are the elastic $^{12}$ constants for $\mathrm{A} \equiv \mathrm{TbFe}_{2}$ and $\mathrm{B} \equiv \mathrm{YFe}_{2}$. From the data available in the literature [47], we obtain that $\mathcal{K} \simeq 0$ and $\beta_{1} \simeq 0.7$. In Fig. $18, a$ we present the RT values of $b_{1}$ for our bilayers and for bulk $\mathrm{TbFe}_{2}, b_{1}^{v 0}=-49.5 \mathrm{MPa}[47,48,51]$. Notice that, for the $\mathrm{TbFe}_{2}(300 \AA) / \mathrm{YFe}_{2}(1000 \AA)$ bilayer, $b_{1}$ is negligible compared with the other $b_{1}$ values. Although the value for $t=300 \AA$ could not be experimentally determined, we should notice that $b_{2}$ in this bilayer is rather small and that $b_{1}<b_{2}$ for bulk $\mathrm{TbFe}_{2}$ and for the rest of the bilayers. Again the fit by model Eq. (39) is good in the available region of thickness. Now the fitting parameters are: $d_{1}^{v}\left(+\varepsilon_{0} / 2-e_{0}\right)=-0.32 \mathrm{GPa}$ and $b_{1}^{S}=110 \mathrm{GPa} \cdot \mathrm{A}$. At RT, $+\varepsilon_{0} / 2-e_{0}=-0.16 \%$ and then, $d_{1}^{v}=200 \mathrm{GPa}$. For instance, for $t=1000 \AA$, the different contributions to $b_{1}$ are: $b_{1}^{v}=-0.24 \mathrm{GPa}$ and $b_{1}^{s} / t=0.11 \mathrm{GPa}$, both being of comparable size, but of opposite sign. This is clearly reflected in the minimum exhibited by $b_{1}$ at $t \simeq 1000 \AA$, because of the competition of $b_{1}^{v}$ and $b_{1}^{s}$. For $b_{2}$ both contributions, $b_{2}^{v}$ and $b_{2}^{s}$, have the same sign and, so, the competition is absent. It is also clear that for 


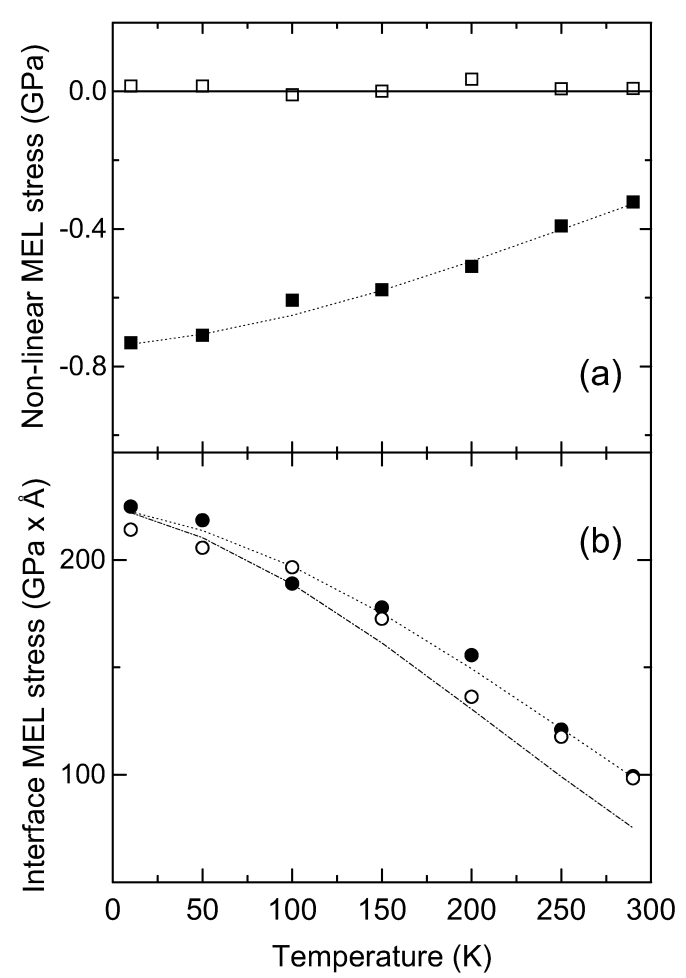

Fig. 19. Thermal dependence of $(a)$ the non-linear MEL stresses: $d_{1}^{v} \varepsilon_{x x}^{0}(\boldsymbol{\square})$ and $d_{2}^{v} \varepsilon_{x y}^{0}(\square)$, and (b) interface MEL stresses: $b_{1}^{s}(\mathbf{\bullet})$ and $b_{2}^{s}(\bigcirc)$. All of them were obtained from the best fit to $b_{1}$ and $b_{2}$. The dotted lines are the fits according to $\hat{I}_{5 / 2}$ Callen and Callen law [27]. The dash-dotted line is the thermal dependence for the $2 D$-spin model [40].

$b_{1}$ the misfit strain strongly affects the bulk's value, $b_{1}^{\vee 0}$, although keeping its sign.

The same kind of fitting was done for the other measurement temperatures. The temperature variations of the fitted parameters $d_{1}^{\mathrm{v}}\left(+\varepsilon_{0} / 2-e_{0}\right)$ and $d_{2}^{v} \varepsilon_{0}$ are shown in Fig. 19,a. Due to the lack of experimental data about $\varepsilon_{0}$, we cannot separate the MEL parameters $d_{1}^{v}$ and $d_{2}^{v}$ for the whole range of temperatures, as we did at RT. The thermal variation of $d_{1}^{v} \varepsilon_{x x}^{0}$ and $d_{2}^{v} \varepsilon_{x y}^{0}$ is rather different: $\left|d_{1}^{v} \varepsilon_{x x}^{0}\right|$ decrease with increasing temperature and $\left|d_{2}^{v} \varepsilon_{x y}^{0}\right|$ remains constant. In Fig. 19, $b$ we show the thermal variation of the interface MEL parameters $b_{1}^{s}$ and $b_{2}^{s}$. Noticeable is that $b_{1}^{s}$ and $b_{2}^{s}$ are almost the same, although they are associated to quite different MEL stresses modes, $b_{1}$ (tetragonal stress) and $b_{2}$ (rhombohedral stress). This means that $\partial b_{2} / \partial \varepsilon_{x y}^{0}$ and $\partial b_{1} / \partial \varepsilon_{x x}^{0}$ are quite sensitive to the magnetostriction mode. This is not surprising since both are axial modes.

To ascertain the spin dimensionality, $D$, for the different MEL contributions to the MEL stress parameters we analysed their thermal dependence in terms of Callen and Callen standard theory $[27,40]$ of single-ion crystal electric field magnetostriction.
Thus, for volume spins $(D=3)$ we expect: $b_{i}^{v}(T)=$ $=b_{i}^{v, 0}(T)+\left(d_{i}^{v}(0 \mathrm{~K}) \varepsilon_{i}^{0}(T, t)\right) \hat{I}_{5 / 2}\left[\mathcal{L}^{-1}(m(T))\right] \quad(i=1$, 2 and $\varepsilon_{1}^{0}=\varepsilon_{x x}^{0}, \varepsilon_{2}^{0}=\varepsilon_{x y}^{0}$ ), where $m(T)$ is the reduced magnetization of Tb sublattice $\left(m(T)=M_{\mathrm{Tb}}(T) / M_{\mathrm{Tb}}(0)\right)$; $\mathcal{L}^{-1}$ is the inverse Langevin function; $\hat{I}_{5 / 2}$ is the Bessel reduced function of first kind. In Fig. 19, $a$ we plot the thermal dependences of $d_{1}^{v} \varepsilon_{x x}^{0}$ and $d_{2}^{v} \varepsilon_{x y}^{0}$ and the fit by the above formula, where we have used the values of $b_{i}^{v, 0}(T)$ existing in the literature for bulk $\mathrm{TbFe}_{2}$ [47-51]. The agreement is good, indicating Heisenberg spins.

On the other hand, for the interfacial MEL stresses we tried two scalings: $b_{i}^{s}(T)=b_{i}^{s}(0 \mathrm{~K}) m^{\alpha}(T)$ $(i=1,2)$ with $\alpha=4$ at low temperatures and $\alpha=2$ in the high temperature range. This is the scaling predicted for spin dimension $D=2$; a scaling with $\alpha=3$ for low temperatures indicates an spin dimension $D=3$.

The reduced magnetization, $m(T)$, was determined by subtracting from the $M_{s, \mathrm{TbFe}_{2}}(T)$, the weighted iron sublattice magnetization, which was assumed to be the same as for $\mathrm{YFe}_{2}$ [18]. In Fig. 19,b we plot, as an example, the scaling of the interfacial MEL stress parameter $b_{i}^{s}(T)(i=1,2)$ with $m^{4}(T)$ and $m^{3}(T)$ (notice that the temperatures are sufficiently low and the $m^{2}(T)$ regime does not appear). The agreement is perhaps better with the $m^{3}(T)$ scaling at higher temperatures, but at the lower ones the scalings are equally satisfactory. Therefore it is difficult to decide about the spin dimensionality at the interfaces; and whether the spins are confined or not to the growing plane.

\section{Conclusions}

The relevance of the magnetoelastic energy associated to the interfaces and the epitaxial stress dependence of the volume magnetoelastic stress, which implies a non-linear behavior of the volume magnetoelastic energy, has been investigated by studying the magnetoelastic behavior of two series of Ho-based superlattices. Measurements of the basal plane magnetoelastic stress isotherms in $\mathrm{Ho}_{n_{\mathrm{Ho}}} / \mathrm{Lu}_{15}$ and in $\mathrm{Ho}_{10} / \mathrm{Y}_{n_{\mathrm{Y}}}$ SL's, between $10 \mathrm{~K}$ and above the Néel temperatures, allowed us the determination of interface magnetoelastic stresses which are of the order, even higher, than the volume ones. The modification of the bulk's magnetoelastic stress due to the epitaxial strain is also found to be important in both series. Moreover, the thermal dependence of the magnetoelastic stress is in agreement with a single-ion crystal field character for the magnetoelastic interaction in the studied superlattices. In $\mathrm{Dy} / \mathrm{Y}$ and $\mathrm{Er} / \mathrm{Lu}$ superlattices we have also performed MEL stress measurements, obtaining the 
different MEL stress contributions for the Dy $/ \mathrm{Y}$ samples, but not for the $\mathrm{Er} / \mathrm{Lu}$, where incomplete saturation leads to inconclusive results. The latter situation also takes place for Ho/Tm SL's; nevertheless, MEL stresses clearly show up the competition between the axial and in-plane anisotropies of $\mathrm{Tm}$ and Ho layers. We have measured the magnetoelastic stress in $\operatorname{TbFe}_{2}(t) / \mathrm{YFe}_{2}(1000 \AA$ ) (with $t=300,600,1000$ and $1300 \AA$ ) epitaxial bilayers. The sample with $t=300 \AA$ is the only one which does not show a measurable MEL stress. For the rest of the bilayers, we have been able to determine all the parameters accounting for the different MEL distortions allowed by the cubic symmetry. We have shown that the epitaxial stress strongly modifies the value of the MEL tetragonal stress, which, due to this, increases more than three times its value as compared with the bulk $\mathrm{TbFe}_{2}$ one for a given range of $t$ values. An interface contribution to the MEL stresses has been also determined. As concerns to the origin of both volume and interface contributions, the thermal dependence of the MEL parameters pointed out to a single-ion crystal-electric-field origin for the volume stress contribution and a not well-defined $2 D$ behavior for the spins at the interface.

\section{Acknowledgments}

We acknowledge the financial support of Spanish CICYT under grants MAT95-1539, MAT971038 and CSIC-CNRS project HF1997-0074.

1. R. W. Erwin, J. J. Rhyne, M. B. Salamon, J. A. Borchers, S. Shantanu, R. R. Du, J. E. Cunningham, and C. P. Flynn, Phys. Rev. B35, 6808 (1987); J. A. Borchers, M. B. Salamon, R. W. Erwin, J. J. Rhyne, R. R. Du, and C. P. Flynn Phys. Rev. B43, 3123 (1991); R. S. Beach, J. A. Borchers, A. Matheny, R. W. Erwin, M. B. Salamon, B. Everitt, K. Pettit, J. J. Rhyne, and C. P. Flynn, Phys. Rev. Lett. 70, 3502 (1993).

2. C. F. Majkzrak, J. Kwo, M. Hong, Y. Yafet, Doon Gibbs, C. L. Chien, and J. Bohr, Adv. Phys. 40, 99 (1991).

3. D. A. Jehan, D. F. McMorrow, R. A. Cowley, R. C. C. Ward, M. R. Wells, N. Hagmann, and K. N. Clausen, Phys. Rev. B48, 5594 (1993).

4. M. Ciria, J. I. Arnaudas, A. del Moral, G. J. Tomka, C. de la Fuente, P. A. J. de Groot, M. R. Wells, and R. C. C. Ward, Phys. Rev. Lett. 75, 1634 (1995).

5. A. del Moral, M. Ciria, J. I. Arnaudas, R. C. C. Ward, and M. R. Wells, J. Appl. Phys. 81, 5311 (1997).

6. J. I. Arnaudas, A. del Moral, M. Ciria, C. de la Fuente, R. C. C. Ward, and M. R. Wells, in: Frontiers in Magnetism of Reduced Dimension Systems, V. G. Baryakhtar, P. E. Wigen, and N. A. Lesnik (eds.), Kluwer Acad. Publishers, Dordrecht, pp. 525-552 and References therein (1998).

7. A. del Moral, and E. W. Lee, J. Phys. C: Solid State Phys. 8, 3881 (1975).
8. P. P. Swaddling, D. F. McMorrow, J. A. Simpson, M. R. Wells, R. C. C. Ward, and K. N. Clausen, J. Phys.: Condens. Matter 5, L481 (1993).

9. T. Honda, Y. Hayashi, K. I. Arai, K. Ishiyama, and M. Yamaguchi, IEEE Trans. Magn. MAG-29, 3126 (1993); ibid. p. 3129.

10. P. I. Williams, D. G. Lord, and P. J. Grundy, J. Appl. Phys. 75, 5257 (1994).

11. E. Quandt, J. Appl. Phys. 75, 5653 (1994).

12. T. Honda, K. I. Arai, and M. Yamaguchi, J. Appl. Phys. 76, 6994 (1994).

13. P. J. Grundy, D. G. Lord, and P. I. Williams, J. Appl. Phys. 76, 7003 (1994).

14. N. H. Duc, K. Mackay, J. Betz, and D. Givord, J. Appl. Phys. 79, 973 (1996).

15. E. Quandt, A. Ludwig, J. Betz, K. Mackay, and D. Givord, J. Appl. Phys. 81, 5420 (1997).

16. V. Oderno, C. Dufour, K. Dumesnil, P. H. Mangin, and G. J. Marchal, J. Crystal Growth 165, 175 (1996).

17. M. Ciria, J. I. Arnaudas, C. Dufour, V. Oderno, K. Dumesnil, and A. del Moral, J. Appl. Phys. 81, 5699 (1997).

18. C. de la Fuente, J. I. Arnaudas, M. Ciria, A. del Moral, C. Dufour, A. Mougin, and K. Dumesnil, to be publ. (2000).

19. E. Klokholm, IEEE Trans. Magn. 12, 819 (1976).

20. E. du Trémolet de Lacheisserie, and J. C. Peuzin, J. Magn. Magn. Mater. 136, 136 (1994).

21. E. du Tremolet de Lacheisserie, Phys. Rev. B51, 15925 (1995).

22. L. D. Landau and E. M. Lifshitz, Theory of Elasticity, 2nd ed., Pergamon Press, Oxford (1970).

23. S. Timoshenko and S. Woinowsky-Krieger, Theory of Plates and Shells, 2nd ed., MacGraw-Hill, New York (1959).

24. R. Watts, M. R. J. Gibbs, W. J. Karl, and H. Szymczak, Appl. Phys. Lett. 70, 2607 (1997).

25. V. Iannotti and L. Lanotte, J. Magn. Magn. Mater. 202, 191 (1999) .

26. M. Ciria, J. I. Arnaudas, del A. Moral, M. R. Wells, and R. C. C. Ward, App. Phys. Lett. 72, 2044 (1998).

27. E. R. Callen and H. B. Callen, Phys. Rev. 139, A455 (1965).

28. C. de la Fuente, et al., to be publ.

29. V. Oderno, C. Dufour, K. Dumesnil, Ph. Mangin, G. Marchal, L. Hennet, and G. Patrat, J. Cryst. Growth 165, 175 (1996)

30. A. Mougin, Ph. D. Thesis, Institut National Polytechnique de Lorraine (France) (1999).

31. A. Mougin, private commun.

32. M. Ciria , J. I. Arnaudas, A. del Moral, M. R. Wells, and R. C. C. Ward, App. Phys. Lett. 72, 2044 (1998).

33. M. Ciria, Ph. D. Thesis, Univ. of Zaragoza (1997).

34. J. J. Rhyne, S. Legvold, and E. T. Rodine, Phys. Rev. 154, 266 (1967).

35. J. Jensen and A. R. Mackintosh, Phys. Rev. Lett 64, 2699 (1990).

36. A. del Moral, M. Ciria, J. I. Arnaudas, M. R. Wells, R. C. C. Ward, and C. de la Fuente, J. Phys. : Condens. Matter 10, L139 (1998).

37. J. H. van der Merwe, J. Appl. Rhys. 34, 123 (1963).

38. L. N'eel, J. Phys. Radium 15, 225 (1954).

39. C. H. Lee, H. He, F. J. Lamelas, W. Vavra, C. Uher, and R. Clarke, Phys. Rev. B42, 1066 (1990).

40. E. Callen, J. Appl. Phys. 53, 8139 (1982).

41. A. del Moral, M. Ciria, J. I. Arnaudas, and C. de la Fuente, Phys Rev. B57, R9471 (1998). 
42. W. C. Koehler, J. W. Cable, E. O. Wollan, and M. K. Wilkinson, J. Appl. Phys. 33, 1124 (1962); W. C. Koehler, J. Appl. Phys. 36, 1078 (1965).

43. D. B. Richards and S. Legvold, Phys. Rev. 186, 508 (1969).

44. C. de la Fuente, J. I. Arnaudas, L. Benito, M. Ciria, A. del Moral, R. C. C. Ward, and M. R. Wells, to be publ. (2000).

45. B. Coqblin, The Electronic Structure of Rare-Earth Metals and Alloys: the Magnetic Heavy Rare Earths, Academic, London (1977); J. Jensen and A. R. Mackintosh, Rare Earth Magnetism, Oxford Univ. Press, Oxford (1991).

46. D. A. Jehan, D. F. McMorrow, J. A. Simpson, R. A. Cowley, P. P. Swaddling, and K. N. Clausen, Phys. Rev. B50, 3085 (1994) and References therein.
47. E. Clark, in: Handbook of the Physics and Chemistry of Rare Earth, K. A. Gschneidner and L. Eyring (eds.), North-Holland, Amsterdam, vol. 2 (1982).

48. A. E. Clark, in: Ferromagnetic Materials, E. P. Wohlfarth (ed.) North-Holland, Amsterdam, vol. 1 (1980).

49. E. W. Lee, Proc. Phys. Soc. 72, 249 (1958).

50. E. W. Lee and J. E. L. Bishop, Proc. Phys. Soc. 89, 661 (1966).

51. A. del Moral and A. Vela, Anales de Fisica (Madrid) 265, 72 (1976).

52. A. Mougin, C. Dufour, K. Dumesnil, N. Maloufi, and Ph. Mangin, Phys. Rev. B59, 5950 (1999).

53. J. H. Basson and C. A. B. Ball, Phys. Status Solidi A46, 707 (1978).

54. K. Ha, M. Ciria, R. C. O'Handley, P. W. Stephens, and S. Pagola, Phys. Rev. B60, 13780 (1999). 